\title{
Preheating of the nonminimally coupled inflaton field
}

\author{
Shinji Tsujikawa * \\ Department of Physics, Waseda University, Shinjuku, Tokyo 169-8555, Japan \\ Kei-ichi Maeda ${ }^{1,2 \dagger}$ \\ Department of Physics, Waseda University, Shinjuku, Tokyo 169-8555, Japan \\ Advanced Research Institute for Science and Engineering, \\ Waseda University, Shinjuku, Tokyo 169-8555, Japan \\ Takashi Torii $\ddagger$ \\ Department of Physics, Tokyo Institute of Technology, Meguro, Tokyo 152, Japan
}

(August 30, 2018)

We investigate preheating of an inflaton field $\phi$ coupled nonminimally to a spacetime curvature by the method of Hartree approximations. In the case of a self-coupling inflaton potential $V(\phi)=\lambda \phi^{4} / 4$, the dynamics of preheating changes by the effect of the negative $\xi$. We find that the nonminimal coupling works in two ways. First, since the initial value of inflaton field for reheating becomes smaller with the increase of $|\xi|$, the evolution of the inflaton quanta is delayed for fixed $\lambda$. Second, the oscillation of the inflaton field is modified and the nonadiabatic change around $\phi=0$ occurs significantly. That makes the resonant band of the fluctuation field wider. Especially for strong coupling regimes $|\xi| \gg 1$, the growth of the inflaton fluctuation is dominated by the resonance due to the nonminimal coupling, which leads to the significant enhancement of low momentum modes. Although the final variance of the inflaton fluctuation does not change significantly compared with the minimally coupled case, we have found that the energy transfer from the homogeneous inflaton to created particles efficiently occurs for $\xi \lesssim-60$.

98.80.Cq, 05.70.Fh, 11.15.Kc

\section{INTRODUCTION}

Recently it has been recognized that a postinflationary reheating begins by an explosive particle production which is called preheating [1,2]. As compared with the old theory of reheating [3], preheating is a nonperturbative process by which the energy of the coherently oscillating inflaton field $\phi$ is efficiently transferred to scalar or other particles by parametric resonance. The existence of this stage would drastically change the thermal history of the Universe, and many authors have in-

\footnotetext{
*electronic address:shinji@gravity.phys.waseda.ac.jp

$\dagger$ electronic address:maeda@gravity.phys.waseda.ac.jp

‡electronic address:torii@th.phys.titech.ac.jp
}

vestigated several issues such as baryogenesis at the GUT scale [4], the non-thermal phase transition [5], the formation of topological defects [6], and gravitational waves [7.

There are two typical models of preheating in the chaotic inflation scenario. One of which is the massive inflaton $m^{2} \phi^{2} / 2$ case. In this model, another scalar field $\chi$ coupled to the inflaton field can be resonantly amplified. There are several numerical works by making use of Hartree approximations [9] and fully nonlinear lattice simulations [10,11]. At the linear stage of preheating, the evolution of produced fluctuations can be analyzed analytically by making use of the Mathieu equation [12]. When the coupling constant is large, parametric resonance turns on from the broad resonance regime, and an efficient particle production is possible overcoming the diluting effect by the expansion of the Universe. However, if the inflaton field $\phi$ does not couple to another scalar field $\chi$, the inflaton fluctuation does not grow because the resonant terms are absent in a single field model.

Another model is the self-coupling inflaton $\lambda \phi^{4} / 4$ case. In this case, the fluctuation of the inflaton field is generated even if we do not introduce another scalar field coupled to inflaton. The non-equilibrium scalar field dynamics are studied by fully nonlinear calculations [13], and are also investigated by making use of Hartree or large- $N$ approximations [14 18]. Since the Ricci scalar averaged over several oscillations of the inflaton field vanishes, the system can be reduced to the theory of the Minkowski spacetime. Making use of the properties of the Lamé equation, we can find that the growth rate of the inflaton quanta is not as large as that of the $\chi$ field in the $m^{2} \phi^{2} / 2+g^{2} \phi^{2} \chi^{2} / 2$ model. However, the inflaton fluctuation grows quasi-exponentially and reaches the maximum value $\left\langle\delta \phi^{2}\right\rangle \sim 10^{-7} M_{\mathrm{PL}}^{2}$. In both models, the backreaction effect on the oscillating inflaton field plays an important role to shut off the resonance. Also, a rescattering effect becomes significant at the final stage of preheating.

Generally, it has been assumed that a nonminimal coupling of the inflaton field or other scalar fields can be neg- 
ligible in preheating phase. However, it was first pointed out in the massive inflaton model that the nonminimally coupled $\chi$ field can grow by the oscillating scalar curvature 19. especially when the coupling constant $\xi$ is negative. Later we investigated the structure of resonance including the $\phi-\chi$ coupling where $\chi$ is nonminimally coupled to the scalar curvature [20]. In that model, resonance occurs in the wider range as compared with the minimally coupled $m^{2} \phi^{2} / 2+g^{2} \phi^{2} \chi^{2} / 2$ model, and the final variance of $\chi$-particles can be larger if $\xi \lesssim-4$. In other inflation models such as the higher-curvature gravity, $\chi$-particles can be produced quite efficiently when $|\xi|$ is of order unity 21].

By these works, we can expect that the nonminimal coupling modifies the structure of the resonance also in the inflation model without other fields and makes preheating more efficient. In the massive inflaton model, since $\xi$ is constrained to be small as $|\xi| \lesssim 10^{-3}$ by the observation of the Cosmic Background Explorer (COBE) satellite [22], the evolution of the system will not change so much even if the nonminimal coupling is taken into account. On the other hand, in the $\lambda \phi^{4} / 4$ model, the constraint of $\xi$ is absent for the negative coupling. Especially when $\xi$ is largely negative, the fine-tuning problem of $\lambda$ is relaxed [23,24]. It was pointed out in Ref. [14, 16] that the nonminimal coupling with the inflaton field would not affect the evolution of the inflaton fluctuation because the Ricci scalar averaged over several oscillations of the inflaton vanishes in reheating phase. However, since the Ricci scalar oscillates by the interaction with the homogeneous inflaton field, there is a possibility that the dynamics of preheating would be altered if we consider the nonminimal coupling. In the minimally coupled case, it is known that the energy transfer from the homogeneous inflaton to produced particles is not efficient. In the nonminimally coupled case, however, there is a possibility that enhanced momentum modes become wider and the efficient particle production is realized. It is worth investigating the dynamics of the nonminimally coupled inflaton field, because this would affect the non-thermal phase transition [5], and the formation of topological defects $[6]$.

In this paper, we consider the dynamics of the nonminimally coupled inflaton field in the chaotic inflation model. We suppose that there are no fields other than inflaton. The Hartree approximation is used to estimate the variance of produced fluctuations. We organize our paper as follows. In the next section, we present basic equations of the background and scalar fields. In Sec. III, we study the dynamics of the nonminimally coupled massless inflaton in the inflationary phase, and present the initial values of the inflaton when the reheating stage starts. In Sec. IV, we investigate how the nonminimal coupling changes the evolution of the system in preheating phase. The final variance of the fluctuation is also presented. We give our conclusion and discussion in the final section.

\section{BASIC EQUATIONS}

We investigate a model where an inflaton field $\phi$ is nonminimally coupled with a scalar curvature $R$ :

$$
\mathcal{L}=\sqrt{-g}\left[\frac{1}{2 \kappa^{2}} R-\frac{1}{2}(\nabla \phi)^{2}-V(\phi)-\frac{1}{2} \xi R \phi^{2}\right],
$$

where $\kappa^{2} / 8 \pi \equiv G=M_{\mathrm{PL}}^{-2}$ is Newton's gravitational constant, and $\xi$ is a coupling constant. $V(\phi)$ is a potential of the inflaton field which is described by

$$
V(\phi)=\frac{1}{2} m^{2} \phi^{2}+\frac{1}{4} \lambda \phi^{4}
$$

In this paper, we mainly consider the self-coupling inflaton model.

In the minimally coupled case, the value of the inflaton field at the time $t_{I}$ when the inflation turns on is required $\phi\left(t_{I}\right) \gtrsim 5 M_{\mathrm{PL}}$ in order to solve the cosmological puzzles. Also, the primordial density perturbation observed by the COBE satellite constrains the coupling of the inflaton field as $m \lesssim 10^{-6} M_{\mathrm{PL}}$ for the massive inflaton model, and $\lambda \lesssim 10^{-12}$ for the self-coupling inflaton model respectively.

However, the dynamics of the Universe changes if the nonminimal coupling is taken into account. Futamase and one of the present authors [22] showed that in the case of the positive coupling, $\xi$ is constrained to be small as $\xi \lesssim 10^{-3}$ in both chaotic models. This is due to the constraint that the effective gravitational constant

$$
G_{\mathrm{eff}}=\frac{G}{1-\phi^{2} / \phi_{c}^{2}}, \quad \text { with } \quad \phi_{c}^{2} \equiv \frac{M_{\mathrm{PL}}^{2}}{8 \pi \xi},
$$

must be positive to connect to our present universe, which yields $\phi<\phi_{c}=M_{\mathrm{PL}} / \sqrt{8 \pi \xi}$. The requirement that $\phi_{c}>\phi\left(t_{I}\right)$ leads the constraint: $\xi \lesssim 10^{-3}$.

The Lagrangian in Eq. 2.1) is reduced to the system in the Einstein frame by a conformal transformation

$$
\hat{g}_{\mu \nu}=\Omega^{2} g_{\mu \nu}
$$

where $\Omega^{2} \equiv 1-\xi \kappa^{2} \phi^{2}$. Then we obtain the following equivalent Lagrangian:

$$
\mathcal{L}=\sqrt{-\hat{g}}\left[\frac{1}{2 \kappa^{2}} \hat{R}-\frac{1}{2} F^{2}(\hat{\nabla} \phi)^{2}-\hat{V}(\phi)\right],
$$

where

$$
F^{2} \equiv \frac{1-(1-6 \xi) \xi \kappa^{2} \phi^{2}}{\left(1-\xi \kappa^{2} \phi^{2}\right)^{2}}
$$

and

$$
\hat{V}(\phi) \equiv \frac{V(\phi)}{\left(1-\xi \kappa^{2} \phi^{2}\right)^{2}}
$$

Introducing a new scalar field $\psi$ as 


$$
\psi \equiv \int F(\phi) d \phi
$$

the Lagrangian in the new frame is reduced to the canonical form:

$$
\mathcal{L}=\sqrt{-\hat{g}}\left[\frac{1}{2 \kappa^{2}} \hat{R}-\frac{1}{2}(\hat{\nabla} \psi)^{2}-\hat{V}(\psi)\right] .
$$

When $\xi$ is negative, the effective potential (2.7) shows a different characteristic from the minimally coupled case. In the massive inflaton case, since the potential has a maximum at $\phi=M_{\mathrm{PL}} / \sqrt{8 \pi|\xi|}$ (See Fig. 1), $\xi$ is constrained to be small as $|\xi| \lesssim 10^{-3}$. As long as we do not introduce another field coupled to inflaton, the fluctuation of the inflaton quanta is not generated in the minimally coupled case. Hence the situation does not change even if we take into account such small values of $|\xi|$.

However, such a constraint is absent in the case of the $\lambda \phi^{4} / 4$ model, because the effective potential of inflaton has a plateau to lead a sufficient inflation (Fig. 1). Especially when the coupling is strong as $|\xi| \gtrsim 1$, several authors 23 25] investigated the dynamics of the nonminimally coupled inflaton field in the inflationary phase. Hereafter, we consider the model of self-coupling potential $V(\phi)=\lambda \phi^{4} / 4$ with negative $\xi$.

Let us obtain the basic equations in the self-coupling model with the nonminimal coupling. We can derive the equivalent equations in both frames of (2.1) and (2.9). However, the equations in both frames are different each other if we take the mean field approximation in each frame. Generally, it is convenient to consider the evolution of the system in the Einstein frame. However, since the conformal factor is included in the denominator of the potential (2.7), we can not replace the mean field value of $\left\langle 1 /\left(1-\xi \kappa^{2} \phi^{2}\right)^{2}\right\rangle$ as $1 /\left(1-\xi \kappa^{2}\left\langle\phi^{2}\right\rangle\right)^{2}$. If these replacements were done, we need further assumptions of the derivative terms of the $\phi$ field in order to coincide with the basic equations of which the mean field approximation is taken in the original frame. Although we expect that these derivative terms do not affect the preheating dynamics significantly, there still remains the ambiguity whether these assumptions are always valid.

Moreover, the conformal factor $\Omega^{2}$ includes quantum variable $\phi^{2}$ since we consider the quantum fluctuation of the $\phi$ field. The metric in the Einstein frame is perturbed by making a conformal transformation even if the metric in the original frame is spatially homogeneous. The analysis of other preheating models including the metric perturbation are performed by several authors [27, but in the present model, there remains the difficulty of how we take the mean field approximation in the Einstein frame. In order to avoid these problems which are accompanied by a conformal transformation, we consider the basic equations in the original frame and take the mean field approximation.

We adopt the flat Friedmann-Robertson-Walker metric as the background spacetime;

$$
d s^{2}=-d t^{2}+a^{2}(t) d \mathbf{x}^{2}
$$

where $a(t)$ is the scale factor. Although we do not consider the metric perturbation in this paper, we should include this effect for a complete study of preheating. It is under consideration.

We obtain the following field equations from the Lagrangian (2.1),

$$
\begin{gathered}
\frac{1-\xi \kappa^{2} \phi^{2}}{\kappa^{2}} G_{\mu \nu}=(1-2 \xi) \nabla_{\mu} \phi \nabla_{\nu} \phi-\left(\frac{1}{2}-2 \xi\right) g_{\mu \nu}(\nabla \phi)^{2} \\
-g_{\mu \nu} V(\phi)+2 \xi \phi\left(g_{\mu \nu} \square-\nabla_{\mu} \nabla_{\nu}\right) \phi \\
\square \phi-\xi R \phi-V,_{\phi}=0,
\end{gathered}
$$

where $\square$ and $V,_{\phi}$ are defined as $\square \equiv \partial_{\mu}\left(\sqrt{-g} g^{\mu \nu} \partial_{\nu}\right) / \sqrt{-g}, V,_{\phi} \equiv \partial V / \partial \phi$ respectively.

Since we consider the quantum fluctuation of inflaton, the $\phi$ field is represented by the homogeneous and fluctuational parts as

$$
\phi(t, \mathbf{x})=\phi_{0}(t)+\delta \phi(t, \mathbf{x}) .
$$

As for the fluctuation part, we impose the tadpole condition

$$
\langle\delta \phi(t, \mathbf{x})\rangle=0
$$

where $\langle\cdots\rangle$ denotes the expectation value. We also make the Hartree factorization as

$$
\begin{array}{r}
(\delta \phi)^{3} \rightarrow 3\left\langle(\delta \phi)^{2}\right\rangle(\delta \phi), \\
(\delta \phi)^{4} \rightarrow 6\left\langle(\delta \phi)^{2}\right\rangle(\delta \phi)^{2}-3\left\langle(\delta \phi)^{2}\right\rangle^{2} .
\end{array}
$$

Then we obtain the Hamiltonian constraint equation by taking the mean field approximation in Eq. (2.11),

$$
\begin{aligned}
H^{2}= & \frac{\kappa^{2}}{3(1-\alpha)}\left[\frac{1}{2}\left(\dot{\phi}_{0}^{2}+\left\langle\delta \dot{\phi}^{2}\right\rangle\right)+\left(\frac{1}{2}-2 \xi\right)\left\langle\delta \phi^{\prime 2}\right\rangle\right. \\
& +\frac{1}{4} \lambda\left(\phi_{0}^{4}+6 \phi_{0}^{2}\left\langle\delta \phi^{2}\right\rangle+3\left\langle\delta \phi^{2}\right\rangle^{2}\right) \\
& \left.+2 \xi\left\{3 H\left(\phi_{0} \dot{\phi}_{0}+\langle\delta \phi \delta \dot{\phi}\rangle\right)-\left\langle\delta \phi \delta \phi^{\prime \prime}\right\rangle\right\}\right]
\end{aligned}
$$

where a dot and a prime denote the derivative with respect to time and space coordinates, respectively, and $\alpha \equiv \xi \kappa^{2}\left\langle\phi^{2}\right\rangle, H \equiv \dot{a} / a$.

Next, we consider the equations of the homogeneous and fluctuational parts of the inflaton field. Let us first obtain the expression of the scalar curvature $R$. Since $R$ can be written by $R=-g^{\mu \nu} G_{\mu \nu}$, we easily find the following relation from Eq. (2.11) as

$$
\frac{1-\xi \kappa^{2} \phi^{2}}{\kappa^{2}} R=(1-6 \xi)(\nabla \phi)^{2}+4 V(\phi)-6 \xi \phi \square \phi .
$$

Then $R$ is expressed as 


$$
\begin{aligned}
R= & \frac{\kappa^{2}}{1-\alpha}\left[(1-6 \xi)\left(-\dot{\phi}_{0}^{2}-\left\langle\delta \dot{\phi}^{2}\right\rangle+\left\langle\delta \phi^{\prime 2}\right\rangle\right)\right. \\
& +\lambda\left(\phi_{0}^{4}+6 \phi_{0}^{2}\left\langle\delta \phi^{2}\right\rangle+3\left\langle\delta \phi^{2}\right\rangle^{2}\right)+6 \xi\left\{\phi_{0} \ddot{\phi}_{0}\right. \\
& \left.\left.+\langle\delta \phi \delta \ddot{\phi}\rangle+3 H\left(\phi_{0} \dot{\phi}_{0}+\langle\delta \phi \delta \dot{\phi}\rangle\right)-\left\langle\delta \phi \delta \phi^{\prime \prime}\right\rangle\right\}\right] .
\end{aligned}
$$

Taking the mean field approximation of Eq. (2.12), the $\phi_{0}$ and $\delta \phi$ fields obey the following equations of motion,

$$
\begin{gathered}
\ddot{\phi}_{0}+3 H \dot{\phi}_{0}+\lambda \phi_{0}\left(\phi_{0}^{2}+3\left\langle\delta \phi^{2}\right\rangle\right)+\xi R \phi_{0}=0, \\
\delta \ddot{\phi}+3 H \delta \dot{\phi}-\partial_{i} \partial^{i}(\delta \phi)+\left\{3 \lambda\left(\phi_{0}^{2}+\left\langle\delta \phi^{2}\right\rangle\right)+\xi R\right\} \delta \phi=0 .
\end{gathered}
$$

Note that both evolutions of the $\phi_{0}$ and $\delta \phi$ fields can be altered by the effect of the nonminimal coupling.

In order to study a quantum particle creation, we expand $\delta \phi$ as

$$
\delta \phi=\frac{1}{(2 \pi)^{3 / 2}} \int\left(a_{k} \delta \phi_{k}(t) e^{-i \mathbf{k} \cdot \mathbf{x}}+a_{k}^{\dagger} \delta \phi_{k}^{*}(t) e^{i \mathbf{k} \cdot \mathbf{x}}\right) d^{3} \mathbf{k},
$$

where $a_{k}$ and $a_{k}^{\dagger}$ are the annihilation and creation operators respectively. Then, we find that $\delta \phi_{k}$ obeys the following equation of motion

$$
\delta \ddot{\phi}_{k}+3 H \delta \dot{\phi}_{k}+\left[\frac{k^{2}}{a^{2}}+3 \lambda\left(\phi_{0}^{2}+\left\langle\delta \phi^{2}\right\rangle\right)+\xi R\right] \delta \phi_{k}=0 .
$$

The expectation values of $\delta \phi^{2}$ and $\phi^{2}$ are expressed as 14.

$$
\begin{aligned}
\left\langle\delta \phi^{2}\right\rangle & =\frac{1}{2 \pi^{2}} \int k^{2}\left|\delta \phi_{k}\right|^{2} d k, \\
\left\langle\phi^{2}\right\rangle & =\phi_{0}^{2}+\left\langle\delta \phi^{2}\right\rangle .
\end{aligned}
$$

Introducing a conformal time $\eta \equiv \int a^{-1} d t$ and new scalar fields $\varphi_{0} \equiv a \phi_{0}$ and $\delta \varphi_{k} \equiv a \delta \phi_{k}$, Eqs. (2.20) and (2.21) are rewritten by

$$
\frac{d^{2} \varphi_{0}}{d \eta^{2}}+\lambda \varphi_{0}\left(\varphi_{0}^{2}+3 a^{2}\left\langle\delta \phi^{2}\right\rangle\right)+\left(\xi R a^{2}-\frac{1}{a} \frac{d^{2} a}{d \eta^{2}}\right) \varphi_{0}=0,
$$

$$
\frac{d^{2}}{d \eta^{2}} \delta \varphi_{k}+\omega_{k}^{2} \delta \varphi_{k}=0
$$

with

$$
\omega_{k}^{2} \equiv k^{2}+3 \lambda\left(\varphi_{0}^{2}+a^{2}\left\langle\delta \phi^{2}\right\rangle\right)+\xi R a^{2}-\frac{1}{a} \frac{d^{2} a}{d \eta^{2}} .
$$

In the case of the minimal coupling, the $3 \lambda \varphi_{0}^{2}$ term in the time dependent frequency (2.28) causes the resonant growth of the inflaton fluctuation. In the nonminimally coupled case, $\xi R a^{2}$ term also contributes to the parametric resonance. In this paper, we mainly investigate how the inflaton fluctuation $\left\langle\delta \phi^{2}\right\rangle$ grows by taking into account the effect of the nonminimal coupling.

As for the initial conditions of the fluctuation, we choose the state that corresponds to the conformal vacuum as

$$
\begin{array}{r}
\delta \varphi_{k}(0)=\frac{1}{\sqrt{2 \omega_{k}(0)}}, \\
\delta \dot{\varphi}_{k}(0)=-i \omega_{k}(0) \delta \varphi_{k}(0) .
\end{array}
$$

We investigate the evolution of the inflaton quanta with those initial conditions as a semiclassical problem. Before proceeding the particle creation in preheating phase, we summarize the evolution of the scale factor and the inflaton field in the inflationary period in the case of the strong coupling in the next section.

\section{THE DYNAMICS OF INFLATION WITH THE NONMINIMAL COUPLING}

In this section, we analyze how inflation takes place in the presence of the nonminimal coupling term. In the inflationary stage, since the fluctuation of the inflaton field is small compared with the homogeneous part, we can approximately write Eqs. (2.17), (2.18), and (2.20) as follows

$$
H^{2}=\frac{\kappa^{2}}{3(1-\alpha)}\left[\frac{1}{2} \dot{\phi}_{0}^{2}+\frac{1}{4} \lambda \phi_{0}^{4}+6 \xi H \phi_{0} \dot{\phi}_{0}\right],
$$

$$
\begin{gathered}
R=\frac{\kappa^{2}}{1-\alpha}\left[-(1-6 \xi) \dot{\phi}_{0}^{2}+\lambda \phi_{0}^{4}+6 \xi \phi_{0}\left(\ddot{\phi}_{0}+3 H \dot{\phi}_{0}\right)\right], \\
\ddot{\phi}_{0}+3 H \dot{\phi}_{0}+\lambda \phi_{0}^{3}+\xi R \phi_{0}=0
\end{gathered}
$$

where $\alpha=\xi \kappa^{2} \phi_{0}^{2}$. By Eq. (3.2), the scalar curvature is eliminated to give

$$
\ddot{\phi}_{0}+3 H \dot{\phi}_{0}+\frac{\lambda \phi_{0}^{3}}{1-(1-6 \xi) \alpha}-\frac{\xi \kappa^{2}(1-6 \xi) \dot{\phi}_{0}^{2}}{1-(1-6 \xi) \alpha} \phi_{0}=0 .
$$

Imposing the slow roll approximations $\left|\ddot{\phi}_{0} / \dot{\phi}_{0}\right| \ll H$, $\left|\dot{\phi}_{0} / \phi_{0}\right| \ll H$, and $\frac{1}{2} \dot{\phi}_{0}^{2} \ll V(\phi)$, Eqs. (3.1) and (3.4) can be written as

$$
H^{2}=\frac{\kappa^{2} \lambda \phi_{0}^{4}}{12(1-\alpha)}\left[1-\frac{8 \xi}{1-(1-6 \xi) \alpha}\right],
$$

$$
3 H \dot{\phi}_{0}=-\frac{\lambda \phi_{0}^{3}}{1-(1-6 \xi) \alpha} .
$$

In order to see the effect of the nonminimal coupling, we consider the strong negative coupling case $\alpha \ll-1$. Then Eqs. (3.5) and (3.6) are simplified as 


$$
\begin{aligned}
H^{2} & =-\frac{\kappa^{2} \lambda \phi_{0}^{4}}{12 \alpha}, \\
3 H \dot{\phi}_{0} & =\frac{\lambda \phi_{0}^{3}}{(1-6 \xi) \alpha} .
\end{aligned}
$$

Combining Eqs. (3.7) and (3.8), we find the following relation

$$
\dot{\alpha}=-\frac{8 \xi}{1-6 \xi} H
$$

This is easily integrated to give

$$
\alpha=\alpha_{I}-\frac{8 \xi}{1-6 \xi} \ln \left(\frac{a}{a_{I}}\right),
$$

where the subscript $I$ denotes the values when the inflationary period starts. We find by this relation that $|\alpha|$ decreases with the expansion of the Universe. Since the scale factor satisfies the relation

$$
\frac{\dot{a}}{a}=\sqrt{\frac{-\lambda \alpha_{I}}{12 \xi^{2} \kappa^{2}}}\left[1-\frac{8 \xi}{(1-6 \xi) \alpha_{I}} \ln \left(\frac{a}{a_{I}}\right)\right]^{1 / 2},
$$

we find that $a$ is expressed as

$$
a=a_{I} \exp \left[H_{I} t-\gamma\left(H_{I} t\right)^{2}\right]
$$

where

$$
H_{I} \equiv \sqrt{\frac{-\lambda \alpha_{I}}{12 \xi^{2} \kappa^{2}}}, \quad \gamma \equiv \frac{2 \xi}{(1-6 \xi) \alpha_{I}} .
$$

At the first stage of inflation, since the $\gamma$ term is negligible, the Universe evolves exponentially. With the passage of time, however, $\gamma$ term becomes important and the expansion rate of the Universe slows down. Fakir and Unruh [23] showed that the inflationary period ends when $|\alpha|$ drops down to unity. This can be easily seen that the term

$$
\left|\frac{\dot{\phi}}{H \phi}\right|=\left|\frac{\dot{\alpha}}{2 H \alpha}\right|=\left|\frac{4 \xi}{(1-6 \xi) \alpha}\right|,
$$

becomes of order unity when $|\alpha| \approx 1$ in the case of $\xi \lesssim-$ 1.

This criterion for the end of the inflation can also be obtained from the effective potential (2.7) in the Einstein frame. The slow-roll parameter of the first order in the Einstein frame is defined by the potential $\hat{V}(\psi)$ as

$$
\epsilon \equiv \frac{1}{2 \kappa^{2}}\left(\frac{\hat{V}^{\prime}(\psi)}{\hat{V}(\psi)}\right)^{2} .
$$

Making use of Eqs. (2.6)-(2.8), this can be written by

$$
\epsilon=\frac{8}{\kappa^{2} \phi_{0}^{2}\left[1-(1-6 \xi) \xi \kappa^{2} \phi_{0}^{2}\right]} .
$$

Setting $\epsilon=1$, the value of $\alpha$ when the inflationary period ends is estimated by

$$
\left|\alpha_{F}\right|=\frac{\sqrt{(1-24 \xi)(1-8 \xi)}-1}{2(1-6 \xi)},
$$

where the subscript $F$ denotes the value at the time $t_{F}$ when the inflation ends. Note that $\left|\alpha_{F}\right|=1$ for $\xi=-1$. $\left|\alpha_{F}\right|$ slowly increases with the decrease of $\xi$ for $\xi<0$. However, since the limiting value for $\xi \rightarrow-\infty$ is $\left|\alpha_{F}\right|=$ 1.15 , inflation ends at $\left|\alpha_{F}\right| \approx 1$ for $\xi \lesssim-1$.

As for the final values of $\phi_{0}$ for the end of inflation, we make use of the ones which are determined by Eq. (3.17) as

$$
\phi_{0}\left(t_{F}\right)=\left[\frac{\sqrt{(1-24 \xi)(1-8 \xi)}-1}{16 \pi(1-6 \xi)|\xi|}\right]^{1 / 2} M_{\mathrm{PL}} .
$$

In the case of the minimal coupling, $\phi_{0}\left(t_{F}\right)=0.56 M_{\mathrm{PL}}$. With the increase of $|\xi|, \phi_{0}\left(t_{F}\right)$ decreases. We show the values of $\left|\alpha_{F}\right|$ and $\phi_{0}\left(t_{F}\right)$ in various cases in TABLE I. Since the large $|\xi|$ makes the inflaton potential flatter, inflation takes place for rather small values of $\phi_{0}$ as compared with the minimal coupling case. As for the time $t_{i}$ at the start of reheating, we assume $t_{i}=t_{F}$. Hence, the initial value of the inflaton field for the preheating is $\phi_{0}\left(t_{i}\right)=\phi_{0}\left(t_{F}\right)$ estimated by Eq. (3.18). In the next section we investigate the preheating dynamics of the nonminimally coupled inflaton field.

\section{PREHEATING OF THE NONMINIMALLY COUPLED INFLATON FIELD}

In this section, we briefly review the minimally coupled inflaton case, and after that, investigate the case that the nonminimal coupling is included.

$$
\text { 1. } \xi=0 \text { case }
$$

In the minimally coupled case, the evolution of the inflaton quanta can be obtained analytically by using the stability and instability chart of the Lamé equation. First, making use of the time-averaged relation $\frac{1}{2}\left\langle\dot{\phi}^{2}\right\rangle_{T}=2\langle V(\phi)\rangle_{T}$, the evolution of the scale factor is approximately written as $a \sim \eta$. Since the scalar curvature is expressed as $R=\frac{6}{a^{3}} \frac{d^{2} a}{d \eta^{2}}$, the time-averaged value of $R$ vanishes in the case of the minimal coupling.

Introducing a dimensionless time variable $x \equiv$ $\sqrt{\lambda} M_{\mathrm{PL}} \eta$ and scalar fields $f \equiv \varphi_{0} / M_{\mathrm{PL}},\left\langle\delta \bar{\phi}^{2}\right\rangle \equiv$ $\left\langle\delta \phi^{2}\right\rangle / M_{\mathrm{PL}}^{2},\left\langle\delta \bar{\varphi}^{2}\right\rangle \equiv a^{2}\left\langle\delta \bar{\phi}^{2}\right\rangle$, Eq. (2.26) is reduced to the equation for the theory $\lambda \phi^{4} / 4$ in Minkowski spacetime:

$$
\frac{d^{2} f}{d x^{2}}+f^{3}\left(1+3 \frac{\left\langle\delta \bar{\varphi}^{2}\right\rangle}{f^{2}}\right)=0
$$


At the stage when the back reaction effect is negligible, the solution of Eq. (4.1) can be handled analytically. Integrating the equation $d^{2} f / d x^{2}+f^{3}=0$, we find

$$
\left(\frac{d f}{d x}\right)^{2}=-\frac{1}{2}\left(f^{4}-f_{\mathrm{co}}^{4}\right),
$$

where $f_{\text {co }}$ is the value when the $\varphi_{0}$ field begins to oscillate coherently. The solution of this equation is expressed by the elliptic cosine,

$$
f=f_{\mathrm{co}} \mathrm{cn}\left(f_{\mathrm{co}}\left(x-x_{\mathrm{co}}\right) ; \frac{1}{\sqrt{2}}\right) .
$$

This function is well approximated as

$$
\begin{aligned}
f & \approx f_{\mathrm{co}} \cos \left(0.8472 f_{\mathrm{co}} x\right) \\
& =f_{\mathrm{co}} \cos \left(0.8472 \sqrt{\lambda} \varphi_{0}\left(t_{\mathrm{co}}\right) \eta\right),
\end{aligned}
$$

where we dropped the initial value of $x_{\text {co }}$ for simplicity. Note that the frequency of the oscillation depends on the values of $\lambda$ and $\varphi_{0}\left(t_{\mathrm{co}}\right)$. As we have showed, initial values of the inflaton for preheating changes if we take into account the effect of the nonminimal coupling. We discuss this issue in the next section.

Since the inflaton field oscillates periodically, the inflaton quanta can be produced by parametric resonance. Eq. (2.27) can be written as

$$
\frac{d^{2}}{d x^{2}} \delta \varphi_{k}+\left[p^{2}+3 f^{2}\left(1+\frac{\left\langle\delta \bar{\varphi}^{2}\right\rangle}{f^{2}}\right)\right] \delta \varphi_{k}=0
$$

where $p^{2} \equiv k^{2} /\left(\lambda M_{\mathrm{PL}}^{2}\right)$ is the normalized momentum. Eq. (4.5) belongs to the class of Lamé equation when the inflaton quanta are not produced significantly. The structures of resonance are studied precisely in Ref. [16, 18]. Making use of the chart of stability and instability bands of the Lamé equation, the growing mode of $\delta \varphi_{k}$ exists in the narrow range:

$$
\frac{3}{2} \tilde{f}^{2}<p^{2}<\sqrt{3} \tilde{f}^{2},
$$

where variables with a tilde $\sim$ means the amplitude of the variables in this paper.

As compared with the model of the massive inflaton plus another scalar field $\chi$, we found that the modes whose momenta are close to $k=0$ are not enhanced and that the maximum growth rate of $\delta \varphi_{k}$ is not so large. In spite of the small growth rate, $\left\langle\delta \phi^{2}\right\rangle$ grows quasi-exponentially by parametric resonance. We show in Fig. 2 the evolution of $\left\langle\delta \phi^{2}\right\rangle$ for the initial value of $\phi_{0}\left(t_{i}\right)=0.56 M_{\mathrm{PL}}$, and the coupling $\lambda=10^{-12}$ which is constrained by the COBE data [26]. At the initial stage, $\left\langle\delta \phi^{2}\right\rangle$ decreases because the expansion rate of the Universe surpasses the particle creation rate. Our numerical calculation based on the mean field approximation reveals that $\left\langle\delta \phi^{2}\right\rangle$ begins to increase after $x \gtrsim 100$, and reaches the maximum value,

$$
\left\langle\delta \phi^{2}\right\rangle_{f}=1.5 \times 10^{-7} M_{\mathrm{PL}}^{2} .
$$

The subscript $f$ denotes the value at the time $t_{f}$ when the $\left\langle\delta \phi^{2}\right\rangle$ reaches the maximum value. This result agrees well with the fully non-linear calculation performed by Khlebnikov and Tkachev [13]. Although our mean field approximation does not include the rescattering effect (i.e. the mode-mode coupling of the inflaton fluctuation), the final variance is consistent with the lattice simulation. This implies that the back reaction effect due to the growth of the inflaton quanta plays an important role for the termination of parametric resonance. As the inflaton fluctuation grows, the back reaction effect prevents the coherent oscillation of the $\varphi_{0}$ field as is found by Eq. (4.1). The resonance band in Eq. (4.5) is shifted away from the original position as particles are produced. According to the analytic estimation by Greene et al. 18], the growth of the inflaton quanta stops when $\left\langle\delta \phi^{2}\right\rangle$ grows up to

$$
\left\langle\delta \phi^{2}\right\rangle_{f} \approx 0.05 \tilde{\phi}_{0}^{2} .
$$

Our numerical value of the termination point is about $x_{f} \approx 440$ and $\tilde{\phi}_{0}\left(x_{f}\right) \approx 1.4 \times 10^{-3} M_{\mathrm{PL}}$, which yields $\left\langle\delta \phi^{2}\right\rangle_{f} \approx 9.8 \times 10^{-8} M_{\mathrm{PL}}^{2}$ by Eq. (4.8). This agrees well with the numerical value of Eq. (4.7). The final fluctuation in the self-coupling inflation model is rather small compared with other preheating models as $m^{2} \phi^{2} / 2+g^{2} \phi^{2} \chi^{2} / 2$ or $m^{2} \phi^{2} / 2+\xi R \chi^{2} / 2$ [12,20]. In the massive inflaton model, preheating starts from the broad resonance regime in certain ranges of the coupling constant. Hence in this case, the growth rate of the fluctuation can take larger values, and the modes which are close to $k=0$ are mainly enhanced. In the present model, however, the instability bands are restricted as Eq. (4.6), and parametric resonance is not so efficient. Parametric resonance ends when the dispersion of the fluctuation becomes about $20 \%$ of the amplitude of the inflaton field. We mainly concern that how the effect of the nonminimal coupling would change the growth of the fluctuation and the final variance. In the next subsection, we investigate this issue precisely.

$$
\text { 2. } \xi \neq 0 \text { case }
$$

Next we proceed to the nonminimally coupled case. Its effect can be seen in several places and changes the dynamics of preheating. As we have already mentioned, the nonminimal coupling alters the initial value of the inflaton field $\phi_{0}$ when the reheating period starts. With the increase of $|\xi|$, this initial value decreases and the frequency of the inflaton oscillation becomes smaller. Second, as is found by Eqs. (2.20) and (2.21), the nonminimal coupling affects the evolutions of the $\phi_{0}$ and $\delta \phi_{k}$ fields through the oscillation of the scalar curvature. At the stage before the inflaton quanta do not grow significantly, the scalar curvature is approximately written by 


$$
R \approx \frac{\kappa^{2}(1-6 \xi)\left(\lambda \phi_{0}^{4}-\dot{\phi}_{0}^{2}\right)}{1-(1-6 \xi) \alpha}
$$

where we used Eqs. (3.2) and (3.3). Note that the oscillation of the scalar curvature depends on $\xi$ significantly. Hereafter, we investigate how the nonminimal coupling would have influence on the evolution of $\phi_{0}$ and $\delta \phi_{k}$ fields. We can rewrite Eqs. (2.26) and (2.27) by taking notice of the relation $R=\frac{6}{a^{3}} \frac{d^{2} a}{d \eta^{2}}$ as

$$
\begin{gathered}
\frac{d^{2} f}{d x^{2}}+f^{3}\left(1+3 \frac{\left\langle\delta \bar{\varphi}^{2}\right\rangle}{f^{2}}\right)+\left(\xi-\frac{1}{6}\right) a^{2} \bar{R} f=0 \\
\frac{d^{2}}{d x^{2}} \delta \varphi_{k}+\left[p^{2}+3 f^{2}\left(1+\frac{\left\langle\delta \bar{\varphi}^{2}\right\rangle}{f^{2}}\right)\right. \\
\left.+\left(\xi-\frac{1}{6}\right) a^{2} \bar{R}\right] \delta \varphi_{k}=0
\end{gathered}
$$

where $\bar{R} \equiv R /\left(\lambda M_{\mathrm{PL}}^{2}\right)$. In the conformal coupling case $(\xi=1 / 6)$, although it breaks the constraint $\xi \lesssim 10^{-3}$, the system is reduced exactly to the equations in Minkowski spacetime.

In the case of the minimal coupling, the last terms in Eqs. (4.10) and (4.11) become negligible after several oscillations of the $\phi_{0}$ field. In the positive $\xi$ case, $\xi$ is constrained to be $\xi \lesssim 10^{-3}$ for the inflationary period to proceed well. We have numerically checked that the evolution of the system is similar to that in the minimally coupled case. Hereafter, we concentrate on the case when $\xi$ is negative and fix the self coupling constant as $\lambda=$ $10^{-12}$. At the end of this section, we will briefly comment the case when $\lambda$ is not fixed.

In the case of $-0.1 \lesssim \xi<0$, since the initial value of $|\alpha|$ is much smaller than unity, the effect of the nonminimal coupling does not alter the evolution of the inflaton significantly compared with the minimally coupled case. When $-10 \lesssim \xi \lesssim-0.1, \xi$ effects begin to be important and change the evolution of the system. In this case, it appears mainly in the value of $\phi_{0}\left(t_{i}\right)$ and $\phi_{0}\left(t_{\text {co }}\right)$. Let us consider the case of $\xi=-1$ as an example. In this case, preheating begins when $\phi_{0}\left(t_{i}\right)=0.1995 M_{\mathrm{PL}}$ and $\left|\alpha_{i}\right|=1$. Since the value of $\phi_{0}\left(t_{\mathrm{co}}\right)$ is small compared with the minimally coupled case, the period of the oscillation of the inflaton field becomes about five times longer than the $\xi=0$ case (See Fig. 3). The growth of the inflaton quanta is delayed due to this effect. Since $|\alpha|$ is of order unity at the end of inflation, we expect that the evolution of the inflaton field and the inflaton quanta would be affected by the presence of the $\left(\xi-\frac{1}{6}\right) a^{2} \bar{R}$ terms in Eqs. (4.10) and 4.11). However, after several oscillations of the inflaton field, $\phi_{0}$ drops down under $0.02 M_{\mathrm{PL}}$, which means that $|\alpha|$ rapidly becomes much smaller than unity. We have numerically checked that these terms are negligible compared with the $f^{3}\left(1+3 \frac{\left\langle\delta \bar{\varphi}^{2}\right\rangle}{f^{2}}\right)$ and $3 f^{2}\left(1+\frac{\left\langle\delta \bar{\varphi}^{2}\right\rangle}{f^{2}}\right)$ terms for $x \gtrsim 100$. Hence the structure of resonance for $x \gtrsim 100$ is almost the same as that in the minimally coupled case.

Since the particle creation rate is smaller than the expansion rate of the Universe at the initial stage of preheating, $\left\langle\delta \phi^{2}\right\rangle$ decreases at the first stage (See Fig. 2). The numerical calculation shows that $\left\langle\delta \phi^{2}\right\rangle$ begins to grow after $x \approx 400$, which means that the evolution of the inflaton quanta is not affected by the $\xi$ effect directly. Although the growth of $\left\langle\delta \phi^{2}\right\rangle$ is delayed due to the decrease of the initial value of the inflaton field, the final variance is almost the same as the $\xi=0$ case. The last term in Eq. (4.11) is completely negligible after $\left\langle\delta \phi^{2}\right\rangle$ reaches to its maximum value, and the final fluctuation can be analyzed in the same way as the minimally coupled case. Numerical values of the maximum point $\left\langle\delta \phi^{2}\right\rangle_{f}=1.7 \times 10^{-7} M_{\mathrm{PL}}^{2}$ and $\tilde{\phi}_{0}\left(x_{f}\right)=1.4 \times 10^{-3}$ are almost the same as those in the minimally coupled case, and parametric resonance terminates before the energy of the $\phi_{0}$ field is sufficiently transferred to the inflaton fluctuation. In this case, the enhanced mode of the momentum exists also in the narrow range which is estimated by Eq. (4.6).

For $\xi \lesssim-10$, the nonminimal coupling becomes so strong that the structure of the resonance changes. Let us first consider the dynamics of preheating in the strong coupling limit: $|\xi| \gg 1$, and later we show the concrete cases.

We assume that the amplitude of the inflaton field is not very small, i.e., $|\xi| \gg \tilde{\alpha} \xi \gg 1$ at the initial stage of preheating, where $\tilde{\alpha}=\xi \kappa^{2} \tilde{\phi}_{0}^{2}$. When the $\phi_{0}$ field oscillates far from the minimum of the potential $\left(\phi_{0} \approx \tilde{\phi}_{0}\right)$, we can approximately express the scalar curvature (4.9) as

$$
\bar{R} \approx \frac{8 \pi}{\alpha}\left(1-\frac{1}{6 \xi \alpha}\right)\left[-\bar{\phi}_{0}^{4}+\frac{1}{a^{2}}\left(\frac{d \bar{\phi}_{0}}{d x}\right)^{2}\right],
$$

where $\bar{\phi}_{0} \equiv \phi_{0} / M_{\mathrm{PL}}$. Here we did not neglect the term $1 /(6 \xi \alpha)$ in Eq. (4.12). This term increases with the passage of time because $|\tilde{\alpha}|$ decreases from the order of unity as long as $\left\langle\delta \phi^{2}\right\rangle \ll \tilde{\phi}_{0}^{2}$. We find from Eq. (4.10) that the equation of the $\phi_{0}$ field is written by

$$
\frac{d^{2} f}{d x^{2}}+\frac{1}{6 \xi}\left(\frac{1}{\alpha}+1\right) f^{3} \approx 0 .
$$

Note that the changing rate of the $f$ field becomes very small around $\phi_{0} \approx \tilde{\phi}_{0}$ in the case of the strong coupling. This behavior is clearly seen in Fig. 4, which shows the evolution of the $\phi$ field in the $\xi=-100$ case.

During one oscillation of the inflaton field, $|(1-6 \xi) \alpha|$ becomes small around the minimum of the potential. In this case, the scalar curvature is approximated as

$$
\bar{R} \approx 48 \pi \xi\left[-\bar{\phi}_{0}^{4}+\frac{1}{a^{2}}\left(\frac{d \bar{\phi}_{0}}{d x}\right)^{2}\right] .
$$

Then the $\phi_{0}$ field satisfies the following equation of motion 


$$
\frac{d^{2} f}{d x^{2}}+\left[1-48 \pi \xi^{2}\left\{\bar{\phi}_{0}^{2}-\frac{1}{a^{2}}\left(\frac{d \bar{\phi}_{0}}{d x}\right)^{2}\right\}\right] f^{3} \approx 0 .
$$

In the very vicinity of $\phi_{0}=0$, Eq. 4.15 ) yields

$$
\frac{d^{2} f}{d x^{2}}+\left[1+48 \pi \xi^{2} \frac{1}{a^{2}}\left(\frac{d \bar{\phi}_{0}}{d x}\right)^{2}\right] f^{3} \approx 0 .
$$

Because of the existence of the last term, the oscillation becomes rapid compared with the minimally coupled case (See Fig. 4). During one oscillation of the inflaton field, the equation changes from (4.13) to (4.15), and from (4.15) to (4.13) as long as the amplitude of the $\phi_{0}$ field satisfies the condition $\tilde{\alpha} \xi \gg 1$. With the passage of time, however, since the amplitude $\tilde{\phi}_{0}$ decreases by the expansion of the universe, the equation of the $\phi_{0}$ field approaches to that of the minimally coupled case.

Next, let us consider the fluctuation of the inflaton field. The particle production occurs when the frequency (2.28) changes nonadiabatically [12]. Since the $\phi_{0}$ field rapidly varies around $\phi_{0} \approx 0$ for the strong coupling $|\xi| \gg 1$, the fluctuation grows nonadiabatically when $\phi_{0}$ is passing through zero. Substituting the relation (4.14) into (4.11), the equation of the fluctuation around $\phi_{0} \approx 0$ is approximately written by 28]

$$
\begin{aligned}
\frac{d^{2}}{d x^{2}} \delta \varphi_{k} & +\left[p^{2}+3 f^{2}-48 \pi a^{2} \xi^{2}\left\{\bar{\phi}_{0}^{4}\right.\right. \\
- & \left.\left.\frac{1}{a^{2}}\left(\frac{d \bar{\phi}_{0}}{d x}\right)^{2}\right\}\right] \delta \varphi_{k} \approx 0 .
\end{aligned}
$$

In the vicinity of $\phi_{0}=0$, the $48 \pi \xi^{2}\left(\frac{d \bar{\phi}_{0}}{d x}\right)^{2}$ term dominates over other terms. We have numerically checked that this term changes most significantly around $\phi_{0} \approx 0$ and mainly causes the nonadiabatic increase of the fluctuation. The structure of resonance is modified by this effect, and the range of the enhanced momentum modes becomes broader. As was presented by Greene et al. [18], the minimally coupled case (i.e. the prefactor of $f^{2}$ is 3 ) is the least favorable case for an efficient preheating. In the present model, however, the effect of the nonminimal coupling makes the resonance band wider and we can expect the rapid growth of the fluctuation.

Let us consider the case of $\xi=-20$. In this case, initial values for preheating are $\phi_{0}\left(t_{i}\right)=0.0477 M_{\mathrm{PL}}$ and $\left|\alpha_{i}\right|=1.145$ (See Table I). At the initial stage of preheating, since the structure of resonance is dominated by Eq. (4.17) around $\phi_{0}=0$ where the particle production occurs, momentum modes close to $k=0$ can be enhanced. In Fig. 5 we show the evolution of $\omega_{1} \equiv 3 f^{2}\left(1+\frac{\left\langle\delta \bar{\varphi}^{2}\right\rangle}{f^{2}}\right)$ and $\omega_{2} \equiv\left(\xi-\frac{1}{6}\right) a^{2} \bar{R}$ terms in Eq. (4.11). We find that the nonminimally coupled term $\omega_{2}$ has larger amplitude than $\omega_{1}$ at the initial stage. Since the periods of both functions are the same, $\omega_{2}$ dominates over $\omega_{1}$ for the nonadiabatic change around $\phi_{0}=0$. However, this term becomes negligible with the decrease of the scalar curvature. The increase of $\left\langle\delta \phi^{2}\right\rangle$ occurs from $x \approx 4000$, because the particle creation rate can not surpass the expansion rate of the Universe for $x \lesssim 4000$. Since the contribution of $\omega_{2}$ is about $10 \%$ of $\omega_{1}$ at $x \approx 4000$, momentum modes close to $k=0$ are marginally enhanced when $\left\langle\delta \phi^{2}\right\rangle$ begins to increase. After $x \approx 6000$, this contribution becomes less than $5 \%$ and the structure of resonance approaches to the minimally coupled case. Actually we can see in Fig. 6 that the growth rate of $\left\langle\delta \phi^{2}\right\rangle$ becomes a bit small for $x \gtrsim 6000$. Although the range of enhanced $k$ modes is wide at the initial stage of preheating, it becomes narrow gradually as preheating proceeds and only the momentum modes which satisfy the relation (4.6) are enhanced. After $x \approx 6000$, the fluctuation increases up to $\left\langle\delta \phi^{2}\right\rangle_{f}=1.3 \times 10^{-7} M_{\mathrm{PL}}^{2},\left(\right.$ at $\left.x_{f}=1.04 \times 10^{4}\right)$, which is almost the same as the $\xi=0$ case. Although the resonant structure is different at the initial stage, this does not affect the final abundance of the fluctuation significantly. One important property is that the final amplitude of the $\phi_{0}$ field is $\tilde{\phi}_{0}\left(x_{f}\right)=8.0 \times 10^{-4}$, which yields $\left\langle\delta \phi^{2}\right\rangle_{f} \approx 0.2 \tilde{\phi}_{0}^{2}$. This means that the energy of the homogeneous inflaton field is more efficiently transferred to the inflaton fluctuation than in the minimally coupled case.

With the increase of $|\xi|$, the duration of the initial resonant stage caused by the nonminimal coupling continues longer. However, as time passes and $|\alpha|$ decreases, the structure of resonance approaches the minimally coupled case. In the case of $\xi=-50$, the initial value of the inflaton is $\phi_{0}\left(t_{i}\right)=0.0303 M_{\mathrm{PL}}$, which is much smaller than that of the $\xi=0$ case. Because of this, the growth of the inflaton fluctuation is delayed. At the initial stage, low momentum modes can be enhanced, and this is much more efficient than the minimally coupled case with the same initial value of the inflaton. However, for $4000 \lesssim x \lesssim 7000,\left\langle\delta \phi^{2}\right\rangle$ shows a short plateau. This means that the range of enhanced $k$ modes becomes narrow after $x \approx 4000$, and the contribution of low momentum modes becomes less important. For $x \gtrsim 7000$, the higher momenta begin to contribute to the growth of $\left\langle\delta \phi^{2}\right\rangle$. Although we can expect the growth of low momentum modes even for $x \gtrsim 7000$, it is rather inefficient and the fluctuation produced at the final stage is mainly dominated by the higher momenta which are estimated by Eq. (4.6). The final variance is $\left\langle\delta \phi^{2}\right\rangle_{f}=9.5 \times 10^{-8} M_{\mathrm{PL}}^{2}$ at $x_{f}=2.4 \times 10^{4}$. The resonance stops by the back reaction effect of created particles and the final variance does not change so much compared with the $\xi=0$ case. However, since $\tilde{\phi}_{0}\left(x_{f}\right)=4.1 \times 10^{-4}$, we can estimate as $\left\langle\delta \phi^{2}\right\rangle_{f}=0.56 \tilde{\phi}_{0}^{2}$ at the termination point of the resonance. This means that the energy transfer from the $\phi_{0}$ field to $\delta \phi_{k}$ particles becomes more efficient with the increase of $|\xi|$.

We show in Fig. 7 the evolution of the fluctuation for $\xi=-70$ and -80 cases. The structure of the resonance 
is qualitatively same as the $\xi=-50$ case. Since the $\xi$ effect to the fluctuation continues longer with the increase of $|\xi|$, the fluctuation reaches the first plateau at $x=7000$ and 8000 for $\xi=-70$ and -80 cases, respectively. After a short plateau, the secondary resonant stage turns on and $\left\langle\delta \phi^{2}\right\rangle$ begins to increase. However, at this stage, the growth rate is not so large as the first resonant stage, because the enhanced $k$ modes are restricted in the narrow range. We have found that the final variance slowly decreases with the decrease of $\xi$ for $-80 \lesssim \xi \lesssim-50$. For example, when $\xi=-50,-70$, and -80 , the final variances are $\left\langle\delta \phi^{2}\right\rangle_{f} / M_{\mathrm{PL}}^{2}=9.5 \times 10^{-8}$, $7.8 \times 10^{-8}$ and $4.7 \times 10^{-8}$, respectively. As for the ratio of $\left\langle\delta \phi^{2}\right\rangle_{f}$ to $\tilde{\phi}_{0}^{2}$ at the maximum point of the fluctuation, $\left\langle\delta \phi^{2}\right\rangle_{f} / \tilde{\phi}_{0}^{2}=0.56,1.15,1.16$ for $\xi=-50,-70,-80$, respectively. Numerical calculations confirm that resonance stops when the fluctuation grows up to $\left\langle\delta \phi^{2}\right\rangle_{f} \approx \tilde{\phi}_{0}^{2}$ for $\xi \lesssim-60$. Although $\tilde{\phi}_{0}\left(x_{f}\right)$ becomes smaller as $\xi$ decreases, the energy of the oscillating inflaton field is transferred efficiently to the inflaton particles for $\xi \lesssim-60$ as compared with the minimally coupled case. This is mainly due to the fact that nonadiabatic creation of the inflaton particles efficiently occurs around the minimum of the potential.

For $\xi \lesssim-100$, the structure of resonance is dominated by the $\tilde{\xi}$ effect. We show the evolution of the inflaton fluctuation in Fig. 8 for the $\xi=-100,-200$ and -1000 cases. We can not separate the resonant stage into two parts, but find that low momentum modes are enhanced continuously until $\left\langle\delta \phi^{2}\right\rangle$ reaches to its maximum value. Because of the nonexistence of the secondary inefficient resonant stage, the resonance terminates rather early and $\tilde{\phi}_{0}\left(x_{f}\right)$ takes larger value than that in the $-80 \lesssim \xi \lesssim-50$ case. For example, when $\xi=-200, \tilde{\phi}_{0} \approx 1.0 \times 10^{-3}$, which is much larger than the value $\tilde{\phi}_{0} \approx 2.0 \times 10^{-4}$ in the $\xi=-80$ case. This increase of the final value of $\tilde{\phi}_{0}$ results in the increase of the final fluctuation, since resonance ends by the back reaction effect when $\left\langle\delta \phi^{2}\right\rangle$ grows up to the comparable value with $\tilde{\phi}_{0}^{2}$. For example, the final values are $\left\langle\delta \phi^{2}\right\rangle_{f}=2.35 \times 10^{-7} M_{\mathrm{PL}}^{2}$ for $\xi=-100$; $\left\langle\delta \phi^{2}\right\rangle_{f}=1.86 \times 10^{-6} M_{\mathrm{PL}}^{2}$ for $\xi=-200$. For $\xi \lesssim-200$, however, since the final value of $\tilde{\phi}_{0}$ becomes smaller with the decrease of $\xi$ again due to the decrease of the initial value of $\phi_{0}$, the final variance also decreases. For example, $\left\langle\delta \phi^{2}\right\rangle=1.03 \times 10^{-6} M_{\mathrm{PL}}^{2}$ for $\xi=-500$, and $\left\langle\delta \phi^{2}\right\rangle=5.27 \times 10^{-7} M_{\mathrm{PL}}^{2}$ for $\xi=-1000$. In the case of $\xi \lesssim-200$ the nonadiabatic change of the fluctuation in the vicinity of $\phi_{0}=0$ is clearly seen in Fig. 8 . This is because the $\xi R a^{2}$ term in Eq. (2.28) rapidly changes around $\phi_{0}=0$ especially when $|\xi|$ is very large as $|\xi| \gtrsim 200$. However, in this case also, the growth of the fluctuation is finally suppressed by the back reaction effect of created particles.

We have found that the final variance of the nonminimally coupled case does not change significantly compared with the minimally coupled case as is found in
Fig. 9, where the maximum value of $\left\langle\delta \phi^{2}\right\rangle$ is $\left\langle\delta \phi^{2}\right\rangle_{f}=$ $2 \times 10^{-6} M_{\mathrm{PL}}^{2}$ at $\xi \approx-200$. However, the dispersion of the final fluctuation becomes comparable to the amplitude of the $\phi_{0}$ field for $\xi \lesssim-60$ (See Fig. 10). This implies that although the initial value of inflaton decreases as increase of $|\xi|$, this is compensated by the efficient resonance with the appearance of new resonant modes close to $k=0$, We depict in Fig. 11 the distribution of produced momentum modes for the $\xi=0,-1,-50,-100$ cases. With the decrease of $\xi$, we find that momentum modes close to $k=0$ are mainly enhanced. When $\xi \lesssim-100$, the final fluctuation is dominated by low momentum modes, and this is different from the minimally coupled case. As we have seen, preheating stage generically exists even if the nonminimal coupling is taken into account.

Finally, we should mention the case when $\lambda$ is changed. For largely negative $\xi$, larger values of $\lambda$ is allowed to fit the density perturbation because the effective potential (2.7) becomes flat with the increase of $|\xi|$. As is found by Eq. (4.4), the frequency of the $\phi_{0}$ field becomes large with the increase of $\lambda$, which results in the fast growth of the fluctuation. However, $\lambda$ is scaled out in the normalized equations of the $\phi_{0}$ and $\delta \phi_{k}$ fields. $\lambda$ appears only in the regularization of the initial amplitude of the fluctuation. Since the growth of the fluctuation stops by the back reaction effect of created particles, the final variance is almost the same as the case of the fixed $\lambda$, as was suggested in the minimally coupled case in Ref. [13].

\section{CONCLUDING REMARKS AND DISCUSSIONS}

In this paper, we have investigated preheating of the nonminimally coupled inflaton field in the chaotic inflation model. As for the massive inflaton case, since $\xi$ is constrained to be small as $|\xi| \lesssim 10^{-3}$ to lead a sufficient inflation, the evolution of the system is almost the same as the minimally coupled case. In the self-coupling inflaton case, the constraint of $\xi$ is absent for negative $\xi$. Hence we considered mainly this case and investigated how the ordinary picture of preheating is modified by taking into account the $\xi$ effect.

In the minimally coupled case, the equation of the inflaton fluctuation is reduced to the Lamé equation at the linear stage of preheating. Although this model is not so efficient for the development of the resonance compared with the massive inflaton plus another scalar field $\chi$ coupled to inflaton, the fluctuation $\left\langle\delta \phi^{2}\right\rangle$ increases by parametric resonance and reaches to its maximum value $\left\langle\delta \phi^{2}\right\rangle=1.5 \times 10^{-7} M_{\mathrm{PL}}^{2}$. In reheating phase, the timeaveraged scalar curvature vanishes in this model, and the investigation of preheating is reduced to the theory of Minkowski spacetime. Since the instability band is narrow as $3 \tilde{f}^{2} / 2<p^{2}<\sqrt{3} \tilde{f}^{2}$, the resonance is sensitive to the back reaction effect of created particles. The growth of the fluctuation ends at $\left\langle\delta \phi^{2}\right\rangle_{f} \approx 0.05 \tilde{\phi}_{0}^{2}$, which means 
that the dispersion of the fluctuation is only $20 \%$ of the amplitude of the homogeneous inflaton field.

Taking into account the nonminimal coupling with the scalar curvature, the dynamics of preheating can be altered. We found that the $\xi$ effect works in two ways. First, since the initial value of inflaton for preheating becomes lowered as $|\xi|$ increases, and the frequency of the oscillation of the inflaton field is reduced. Hence the growth of the inflaton fluctuation is delayed by this effect for fixed $\lambda$. Second, since frequencies of the both homogeneous and fluctuational parts change due to the nonminimal coupling, the structure of resonance becomes different from the minimally coupled case. In the case of $-0.1 \lesssim \xi<0$, the evolution of the system is almost the same as the minimally coupled case. In $-10 \lesssim \xi \lesssim-0.1$ case, the decrease of the initial value of inflaton mainly delays the evolution of the system. Although the $\xi$ effect changes both frequencies of the $\phi_{0}$ and $\delta \phi_{k}$ fields at the initial stage of preheating, this works only for a short period. When the particle creation rate surpasses the expansion rate of the Universe and $\left\langle\delta \phi^{2}\right\rangle$ begins to increase, the $\xi$ effect on the $\phi_{0}$ and $\delta \phi_{k}$ fields can be negligible. Hence, after that, the system of the evolution is not affected by the $\xi$ effect. The structure of resonance seems almost the same as the minimally coupled case, and produced momentum modes are restricted in a narrow range when $\left\langle\delta \phi^{2}\right\rangle$ reaches to its maximum value. The final variance does not change significantly compared with the minimally coupled case.

As $\xi$ decreases further $(\xi \lesssim-10)$, the initial stage of preheating is dominated by the effect of the nonminimal coupling. The structure of resonance becomes different from that of the $\xi=0$ case, and the wider range of momenta is allowed. Particle creation occurs nonadiabatically in the vicinity of $\phi_{0}=0$. Although the minimally coupled case is the least favorable one for the development of the resonance because of the limited resonant band, such limitation can be removed by taking into account the nonminimal coupling. When $-80 \lesssim \xi \lesssim-10$, the stage of parametric resonance is separated into two stages. At the first stage, the resonance caused by the nonminimal coupling dominates, and momentum modes close to $k=0$ are efficiently enhanced. With the passage of time, the $\xi$ effect onto equations of the $\phi_{0}$ and $\delta \phi_{k}$ fields becomes less important and the system enters the secondary resonant stage. The second stage is the ordinary resonance stage of the minimally coupled case, and the growth rate of the fluctuation is not so large as that of the first stage. It is true that low momentum modes are enhanced, but the final fluctuation is dominated by higher modes described by Eq. (4.6). Although the final variance slowly decreases with the decrease of $\xi$ for $-80 \lesssim \xi \lesssim-10$, the ratio of the final fluctuation $\left\langle\delta \phi^{2}\right\rangle_{f}$ to the square of the amplitude of the $\phi_{0}$ field gets larger. Especially for $\xi \lesssim-60$, we find $\left\langle\delta \phi^{2}\right\rangle_{f} \approx \tilde{\phi}_{0}^{2}$, which implies that the transfer of the energy from the $\phi_{0}$ field to the fluctuation is much more efficient than the minimally coupled case.

When $\xi \lesssim-100$, the growth of fluctuation ends before the secondary resonant stage appears, and parametric resonance is quite efficient. For $-200 \lesssim \xi \lesssim-100$, the final variance increases with the decrease of $\xi$ and $\left\langle\delta \phi^{2}\right\rangle_{f}$ takes the maximum value $\left\langle\delta \phi^{2}\right\rangle_{f}=2 \times 10^{-6} M_{\mathrm{PL}}^{2}$ at $\xi \approx-200$. For $\xi \lesssim-200$, however, $\left\langle\delta \phi^{2}\right\rangle_{f}$ decreases because the initial value of inflaton becomes smaller with the decrease of $\xi$, which yields the smaller amplitude at the termination point of resonance. We also found the relation $\left\langle\delta \phi^{2}\right\rangle_{f} \approx \tilde{\phi}_{0}^{2}$ for $\xi \lesssim-100$, which means that resonance mainly terminates by the back reaction effect when the dispersion of the fluctuation grows up to the comparable value of the amplitude of the $\phi_{0}$ field.

Finally we have to comment on some points. In this paper, we have made heavy use of the Hartree approximation. In taking into account the back reaction effect of created particles, there are other approaches. One of them is to add the stochastic noise term originated by quantum fluctuation to the inflaton equation. This is based on the closed time path formalism in the nonequilibrium quantum field theory [29], and several authors made use of this method in the context of preheating [30]. It is expected that the growth rate of the fluctuation would be changed by taking into account this effect. Other approach is the lattice simulation. Although the final fluctuation of our numerical calculation in the minimally coupled case coincides well with that of the full nonlinear calculation in Ref. [13], the final variance may change in the nonminimally coupled case performed by the lattice simulation. Since the lattice simulation includes the rescattering effect which is absent in the Hartree approximation, the spectrum of enhanced momentum modes would also change by rescattering of created particles. Although we do not present details of the difference in this paper, we should consider preheating by various approaches of the back reaction effect and compare them with the fully nonlinear calculation.

Recently, several authors considered the metric perturbation in reheating phase [27]. As for the self-coupling inflaton model, it was pointed out in Ref. [31] that the metric perturbation is resonantly amplified even if we do not introduce another scalar field coupled to inflaton. In the present model of the nonminimal coupling, since the super Horizon modes can be enhanced by the strong nonminimal coupling, this may alter the spectrum of the density perturbation. It will be interesting to investigate preheating including the metric perturbation, because modification of the spectrum of the density perturbation would result in some important consequences such as the production of primordial black holes. These issues are under consideration. 


\section{ACKOWLEDGEMENTS}

S. T. would like to thank B. A. Bassett and A. Taruya for useful discussions. T. T. is thankful for financial support from the JSPS. This work was supported partially by a Grant-in-Aid for Scientific Research Fund of the Ministry of Education, Science and Culture (No. 09410217 and Specially Promoted Research No. 08102010), by a JSPS Grant-in-Aid (No. 094162), and by the Waseda University Grant for Special Research Projects.

[1] J. Traschen and R. H. Brandenberger, Phys. Rev. D 42, 2491 (1990); Y. Shatanov, J. Trashen, and R. H. Brandenberger, Phys. Rev. D 51, 5438 (1995).

[2] L. Kofman, A. Linde, and A. A. Starobinsky, Phys. Rev. Lett. 73, 3195 (1994).

[3] A. D. Dolgov and A. D. Linde, Phys. Lett. B116, 329 (1982); L. F. Abbott, E.Fahri, and M. Wise, Phys. Lett. B117, 29 (1982).

[4] E. W. Kolb, A. D. Linde, and A. Riotto, Phys. Rev. Lett. 77, 3716 (1996); E. W. Kolb, A. Riotto, and I. I. Tkachev, Phys. Lett. B423, 348 (1998).

[5] L. Kofman, A. Linde, and A. A. Starobinsky, Phys. Rev. Lett. 76, 1011 (1996); I. I. Tkachev, Phys. Lett. B376, 35 (1996); S. Kasuya and M. Kawasaki, Phys. Rev. D 56, 7597 (1997).

[6] S. Khlebnikov, L. Kofman, A. Linde, and I. I. Tkachev, Phys. Rev. Lett. 81, 2012 (1998); I. I. Tkachev, S. Khlebnikov, L. Kofman, A. Linde, Phys. Lett. B440, 262 (1998); S. Kasuya and M. Kawasaki, Phys. Rev. D 58, 083516 (1998).

[7] S. Khlebnikov and I. I. Tkachev, Phys. Rev. D 56, 653 (1997).

[8] B. A. Bassett, Phys. Rev. D 56, 3429 (1997).

[9] S. Khlebnikov and I. I. Tkachev, Phys. Lett. B390, 80 (1997).

[10] S. Khlebnikov and I. I. Tkachev, Phys. Rev. Lett. 79, 1607 (1997).

[11] T. Prokopec and T. G. Roos, Phys. Rev. D 55, 3768 (1997); B. R. Greene, T. Prokopec, and T. G. Roos, Phys. Rev. D 58, 6484 (1997).

[12] L. Kofman, A. Linde, and A. A. Starobinsky, Phys. Rev. D 73, 3258 (1997).

[13] S. Khlebnikov and I. I. Tkachev, Phys. Rev. Lett. 77, 219 (1996).

[14] D. Boyanovsky, H. J. de Vega, R. Holman, D. S. Lee, and A. Singh, Phys. Rev. D 51, 4419 (1995); D. Boyanovsky, M. D' Attanasio, H. J. de Vega, R. Holman, D. S. Lee, and A. Singh, Phys. Rev. D 52, 6805 (1995); D. Boyanovsky, H. J. de Vega, R. Holman, D. S. Lee, and A. Singh, J. F. J. Salgado, Phys. Rev. D 54, 7570 (1996); D. Boyanovsky, D. Cormier, H. J. de Vega, R. Holman, A. Singh, and M. Srednicki, Phys. Rev. D 56, 1939 (1997);
D. Boyanovsky, R. Holman, S. Prem Kumar, Phys. Rev. D 56, 1958 (1997).

[15] J. Baacke, K. Heitmann, and C. Pätzold, Phys. Rev. D 55, 2320 (1997); ibid D 56, 6556 (1997); J. Baacke and C. Pätzold, hep-ph/9906417.

[16] D. I. Kaiser, Phys. Rev. D 53, 1776 (1995); ibid D 56, 706 (1997).

[17] D. T. Son, Phys. Rev. D 54, 3745 (1996).

[18] P. B. Greene, L. Kofman, A. Linde, and A. A. Starobinsky, Phys. Rev. D 56, 6175 (1997).

[19] B. A. Bassett and S. Liberati, Phys. Rev. D 58, 021302 (1998).

[20] S. Tsujikawa, K. Maeda, and T. Torii, Phys. Rev. D 60, 063515 (1999).

[21] S. Tsujikawa, K. Maeda, and T. Torii, hep-ph/9906501, to appear in Phys. Rev. D.

[22] T. Futamase and K. Maeda, Phys, Rev. D 39, 399 (1989).

[23] R. Fakir and W. G. Unruh, Phys, Rev. D 41, 1783 (1990).

[24] N. Makino and M. Sasaki, Prog. Theor. Phys. 86, 103 (1991).

[25] E. Komatsu and T. Futamase, Phys, Rev. D 58, 023004 (1998).

[26] In other papers, the value of $\lambda=10^{-13}$ is often adopted. However, we have confirmed that the difference for these two cases of $\lambda$ is negligibly small and hardly alters our results and discussions.

[27] H. Kodama and T. Hamazaki, Prog. Theor. Phys. 96, 949 (1996); Y. Nambu and A. Taruya, Prog. Theor. Phys. 97, 83 (1997); B. A. Bassett, D. I. Kaiser, and R. Maartens, Phys. Lett. B455, 84 (1999); B. A. Bassett, F. Tamburini, D. I. Kaiser, and R. Maartens, hep-ph/9901319, to appear in Nucl. Phys. B; F. Finelli and R. Brandenberger, Phys. Rev. Lett. 821362 (1999); M. Parry and R. Easther, Phys. Rev. D 59061301 (1999); B. A. Bassett and F. Viniegra, hep-ph/9909353; B. A. Bassett, C. Gordon, R. Maartens, and D. I. Kaiser hep-ph/9909482.

[28] We have also obtained the approximate equation of the fluctuation around $\phi_{0}=\tilde{\phi}_{0}$. This is expressed as $\frac{d^{2}}{d x^{2}} \delta \varphi_{k}+\left(p^{2}+2 f^{2}\right) \delta \varphi_{k} \approx 0$, and the structure of resonance is different from that of the minimally coupled case. In this case, however, since the $\phi_{0}$ field moves very slowly, the nonadiabatic increase of the fluctuation hardly occurs.

[29] E. Calzetta, A. Campos, and E. Verdaguer, Phys. Rev. D 562163 (1997); E. Calzetta and B. L. Hu, Phys. Rev. D 526770 (1995); A. Campos and B. L. Hu, Phys. Rev. D 58, 125021 (1998).

[30] S. A. Ramsey, B. L. Hu, and A. M. Stylianopoulos, Phys. Rev. D 57, 6003 (1998); B. A. Bassett and F. Tamburini, Phys. Rev. Lett. 81, 2630 (1998); B. A. Bassett, Phys. Rev. D 58, 021303 (1998); V. Zanchin, A. Maia Jr., W. Craig, and R. Brandenberger, Phys. Rev. D 57 4651 (1998); V. Zanchin, A. Maia Jr., W. Craig, and R. Brandenberger, Phys. Rev. D 60, 023505 (1999).

[31] M. Parry and R. Easther, hep-ph/9903550. 
TABLE I. The final value of $|\alpha|$ and $\phi_{0}$ when the inflationary period ends in the $\lambda \phi^{4} / 4$ model for negative $\xi$. We find that $\left|\alpha_{F}\right|$ takes the almost constant value $\left|\alpha_{F}\right| \approx 1$ for $\xi<-1$. On the other hand, $\phi_{0}\left(t_{F}\right)$ becomes smaller as $|\xi|$ increases.

\begin{tabular}{r|cc}
\hline$\xi$ & $\left|\alpha_{F}\right|$ & $\phi_{0}\left(t_{F}\right) / M_{\mathrm{PL}}$ \\
\hline 0 & 0 & 0.5642 \\
-0.1 & 0.4606 & 0.4281 \\
-1 & 1.0000 & 0.1995 \\
-10 & 1.1370 & 0.0673 \\
-50 & 1.1511 & 0.0303 \\
-100 & 1.1529 & 0.0215 \\
-1000 & 1.1545 & 0.0068 \\
\hline \hline
\end{tabular}

\section{Figure Captions}

FIG. 1:

The potential in the equivalent system to the nonminimally coupled inflaton. The dotted and solid curves denote the massive inflaton and the massless inflaton case with $\xi=-0.1$ respectively. The potential of the massive inflaton has a local maximum and inflation is difficult to realize unless $|\xi| \lesssim 10^{-3}$. However, the potential of the massless inflaton has a flat plateau to lead a sufficient inflation for negative $\xi$.

FIG. 2:

The evolution of $\left\langle\delta \bar{\phi}^{2}\right\rangle$ as a function of $x$ in $\xi=0$ and $\xi=-1$ cases. In both cases, the fluctuation grows by parametric resonance. When $\xi=-1$, since the initial value of inflaton becomes smaller compared with the $\xi=0$ case, this makes the growth of the fluctuation delayed. However, the final variance $\left\langle\delta \bar{\phi}^{2}\right\rangle \approx 10^{-7}$ is almost the same as the minimally coupled case.

FIG. 3:

The evolution of the homogeneous inflaton field as a function of $x$ in $\xi=0$ and $\xi=-1$ cases. Since the initial value of inflaton for the $\xi=-1$ case is smaller than that of the $\xi=0$ case, the time interval of the oscillation becomes longer.

FIG. 4:

The evolution of the $\phi_{0}$ field as a function of $x$ in the case of $\xi=-100$. The $\phi_{0}$ field slowly changes around the local maximum point of the potential, but nonadiabatically changes in the vicinity of $\phi_{0} \approx 0$.

FIG. 5:

The evolution of $\omega_{1} \equiv 3 f^{2}\left(1+\frac{\left\langle\delta \bar{\varphi}^{2}\right\rangle}{f^{2}}\right)$ and $\omega_{2} \equiv$ $\left(\xi-\frac{1}{6}\right) a^{2} \bar{R}$ as a function of $x$ in the case of $\xi=$ -20 . The effect by the $\omega_{2}$ term is significant at the initial stage of preheating, but it gradually becomes negligible with the passage of time.

FIG. 6:

The evolution of $\left\langle\delta \bar{\phi}^{2}\right\rangle$ as a function of $x$ in $\xi=-20$ and $\xi=-50$ cases. At the initial stage of preheating, the structure of resonance is modified and the range of enhanced momenta becomes wider. However, this stage ends before $\left\langle\delta \bar{\phi}^{2}\right\rangle$ sufficiently increases and the second resonance stage sets in. In both cases of $\xi=$ -20 and $\xi=-50$, the growth rate of the fluctuation becomes smaller after the first resonance stage ends.

FIG. 7: 
The evolution of $\left\langle\delta \bar{\phi}^{2}\right\rangle$ as a function of $x$ in $\xi=-70$ and $\xi=-80$ cases. We can separate the resonance into two stages. The first stage is dominated by the effect of the nonminimal coupling, which connects to the second stage of ordinary minimal coupling. With the decrease of $\xi$, the initial stage becomes longer.

FIG. 8:

The evolution of $\left\langle\delta \bar{\phi}^{2}\right\rangle$ as a function of $x$ in $\xi=-100$, $\xi=-200$ and $\xi=-1000$ cases. In these cases, the fluctuation reaches to its maximum value only by the first efficient resonance stage by the effect of the nonminimal coupling. Therefore, the final fluctuation is dominated by the momenta close to $k=0$.

FIG. 9:

The final value of $\left\langle\delta \bar{\phi}^{2}\right\rangle$ as a function of $|\xi|$ for the negative $\xi$. In the case of $-80 \lesssim \xi \lesssim 0,\left\langle\delta \bar{\phi}^{2}\right\rangle_{f}$ slowly decreases with the increase of $|\xi|$. For $\xi \lesssim-100$, the resonance becomes efficient by the $\xi$ effect, and $\left\langle\delta \bar{\phi}^{2}\right\rangle_{f}$ takes the maximum value $\left\langle\delta \bar{\phi}^{2}\right\rangle_{f} \approx 2 \times 10^{-6}$ at $\xi \approx-200$. However, $\left\langle\delta \bar{\phi}^{2}\right\rangle_{f}$ slowly decreases for $\xi \lesssim-200$ because the growth of the fluctuation is suppressed by the back reaction effect of created particles.

FIG. 10:

The ratio of the final variance $\left\langle\delta \phi^{2}\right\rangle_{f}$ to the square of the amplitude of the $\phi_{0}$ field. This ratio increases with the decrease of $\xi$ for $-50 \lesssim \xi \lesssim 0$, and reaches the plateau $\left\langle\delta \phi^{2}\right\rangle_{f} / \tilde{\phi}_{0}^{2} \approx 1$ for $\xi \lesssim-60$.

FIG. 11: The spectrum of enhanced momentum modes in $\xi=0, \xi=-1, \xi=-50$, and $\xi=-100$ cases. Although the resonance band is narrow in the minimally coupled case, the low momentum modes begin to be enhanced with the decrease of $\xi$. When $\xi \lesssim-100$, the final fluctuation is dominated by low momentum modes. 
Fig. 1

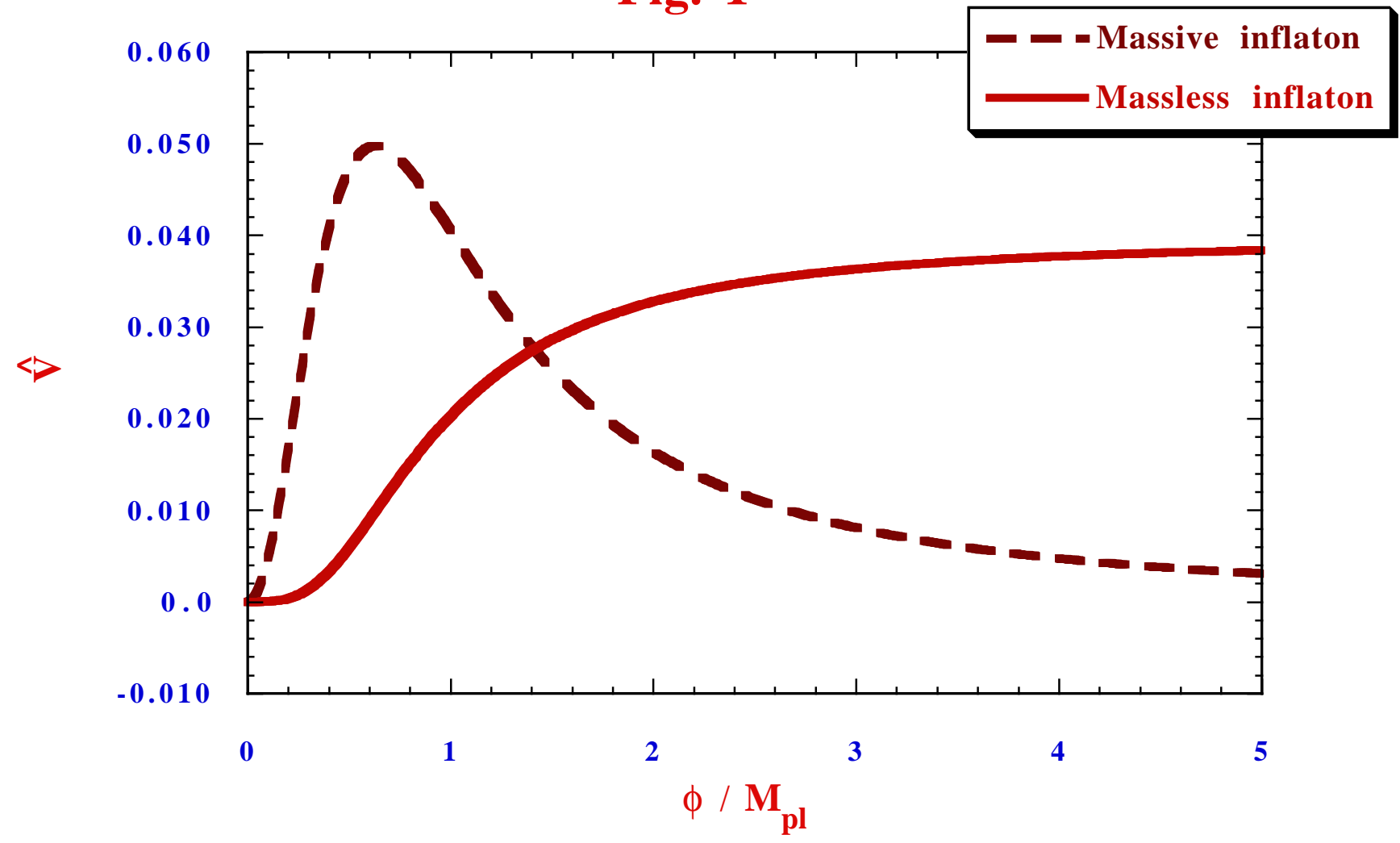


Fig. 2

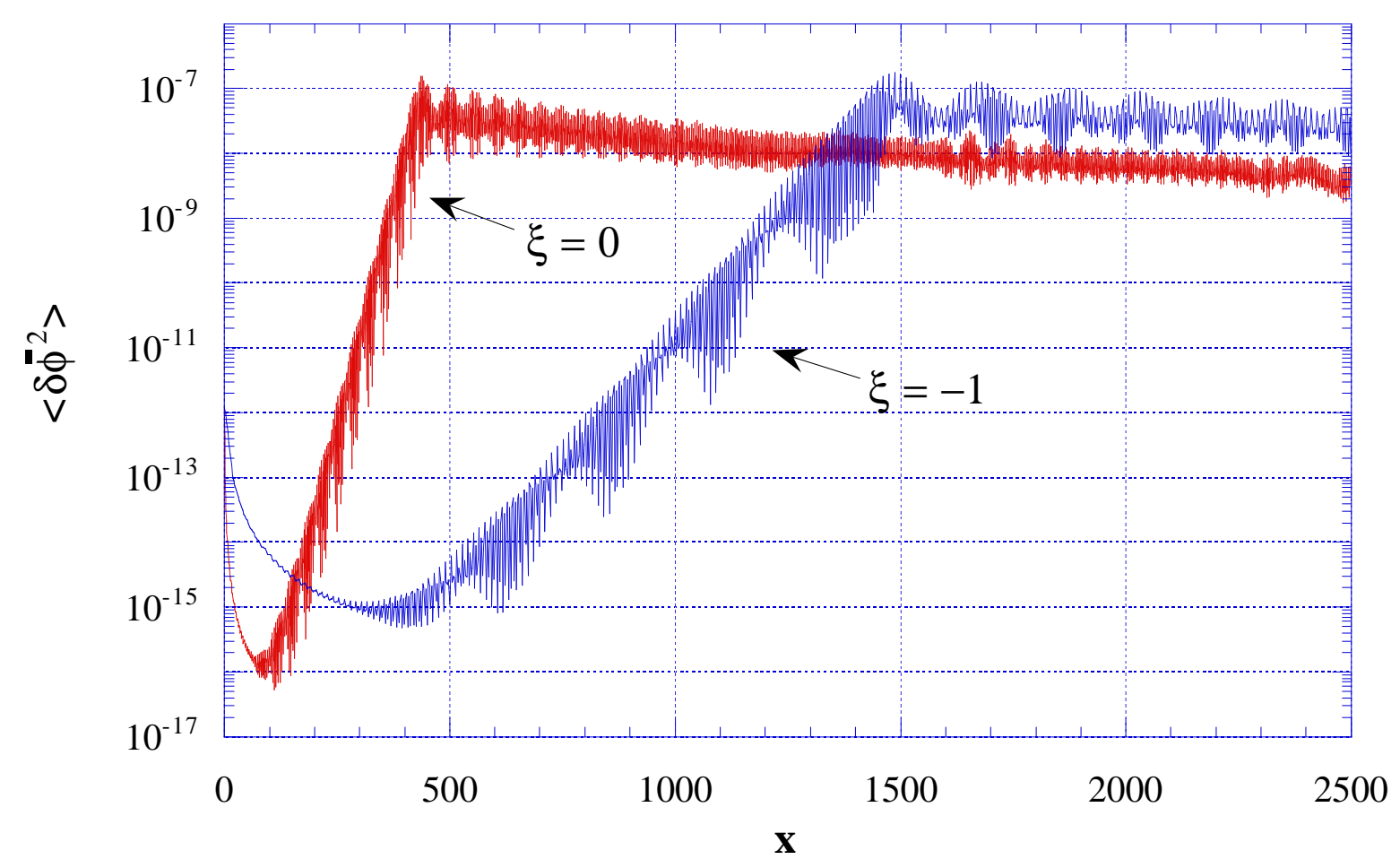


Fig. 3

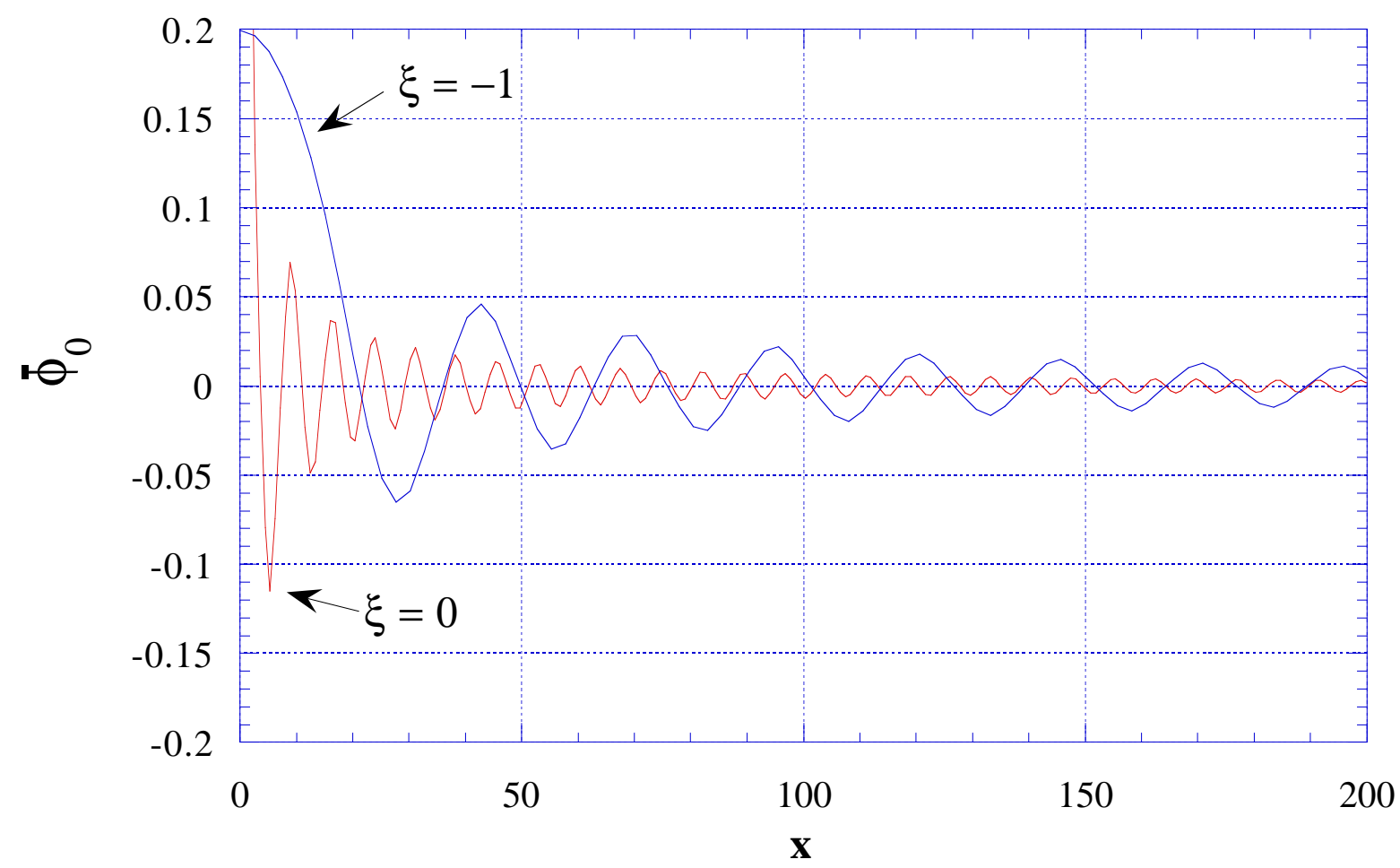


Fig.4

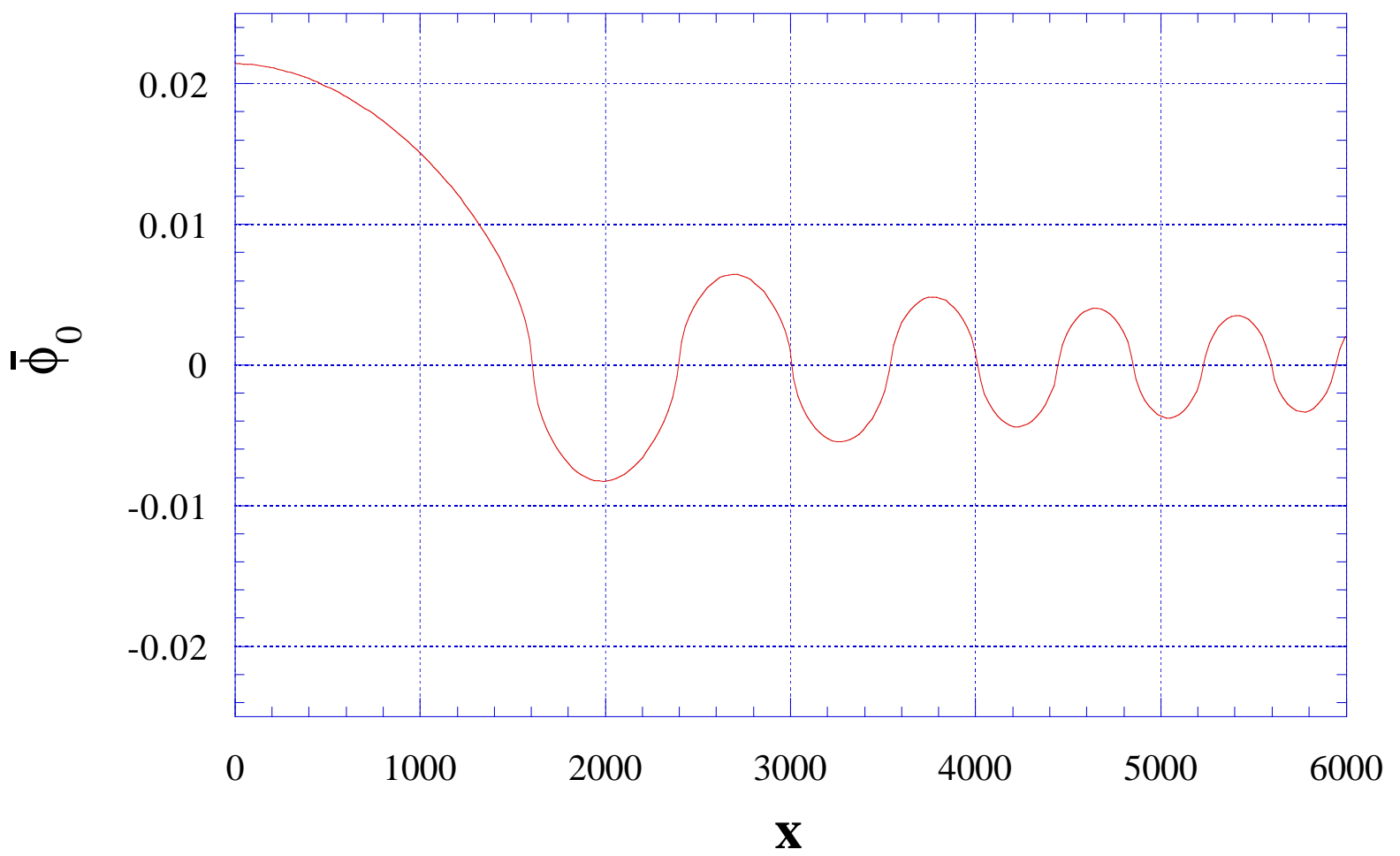


Fig. 5

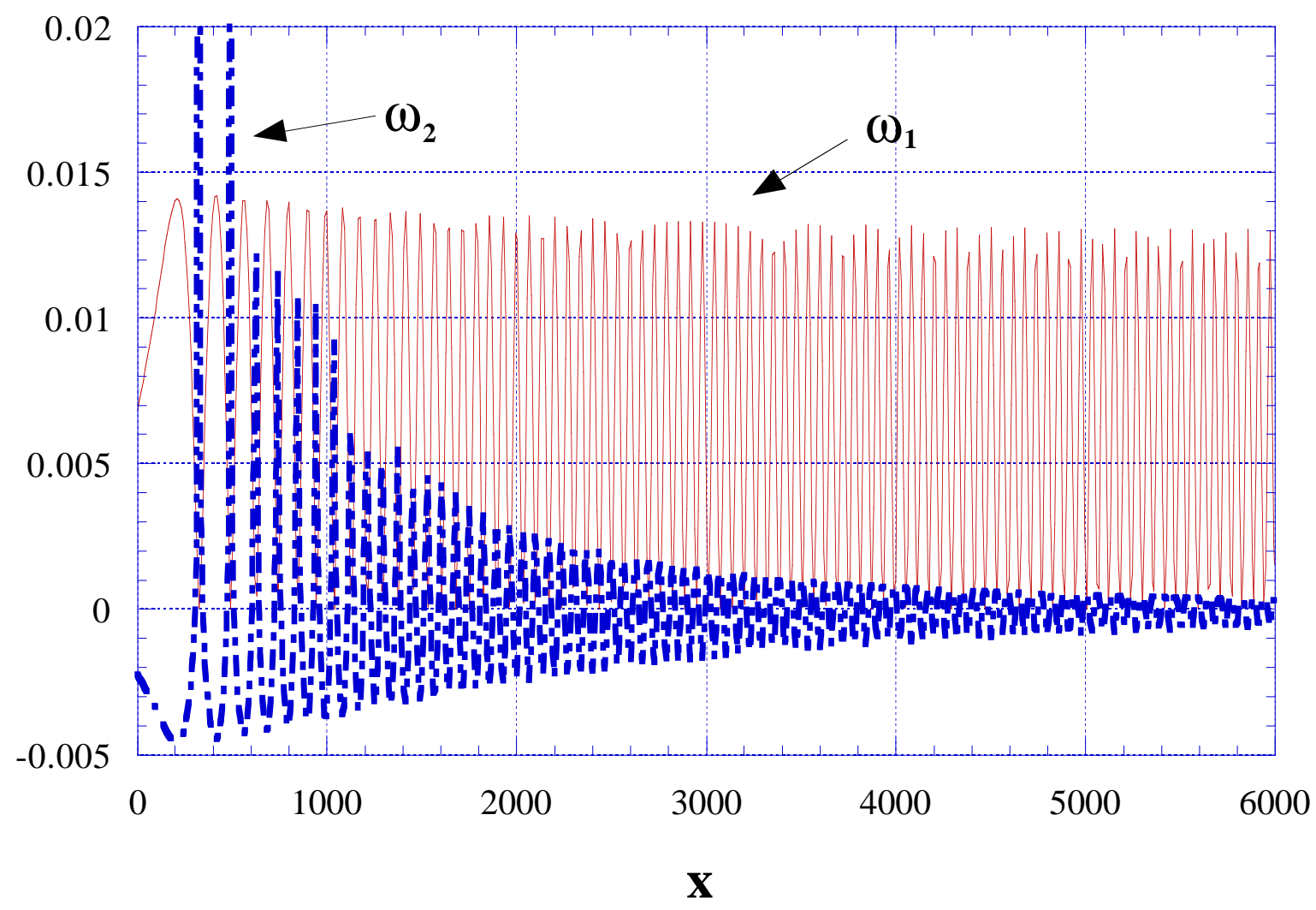


Fig. 6

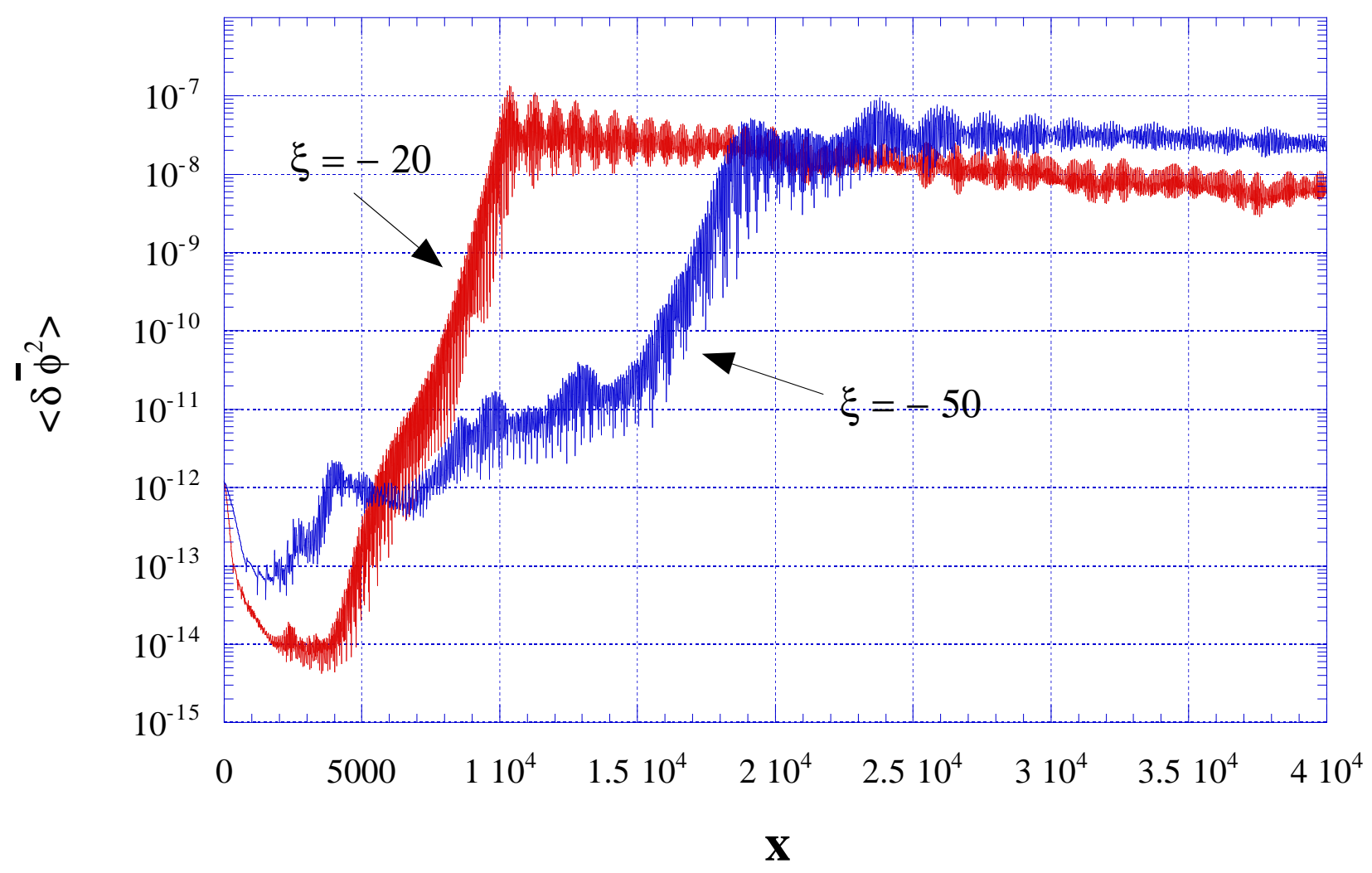


Fig. 7

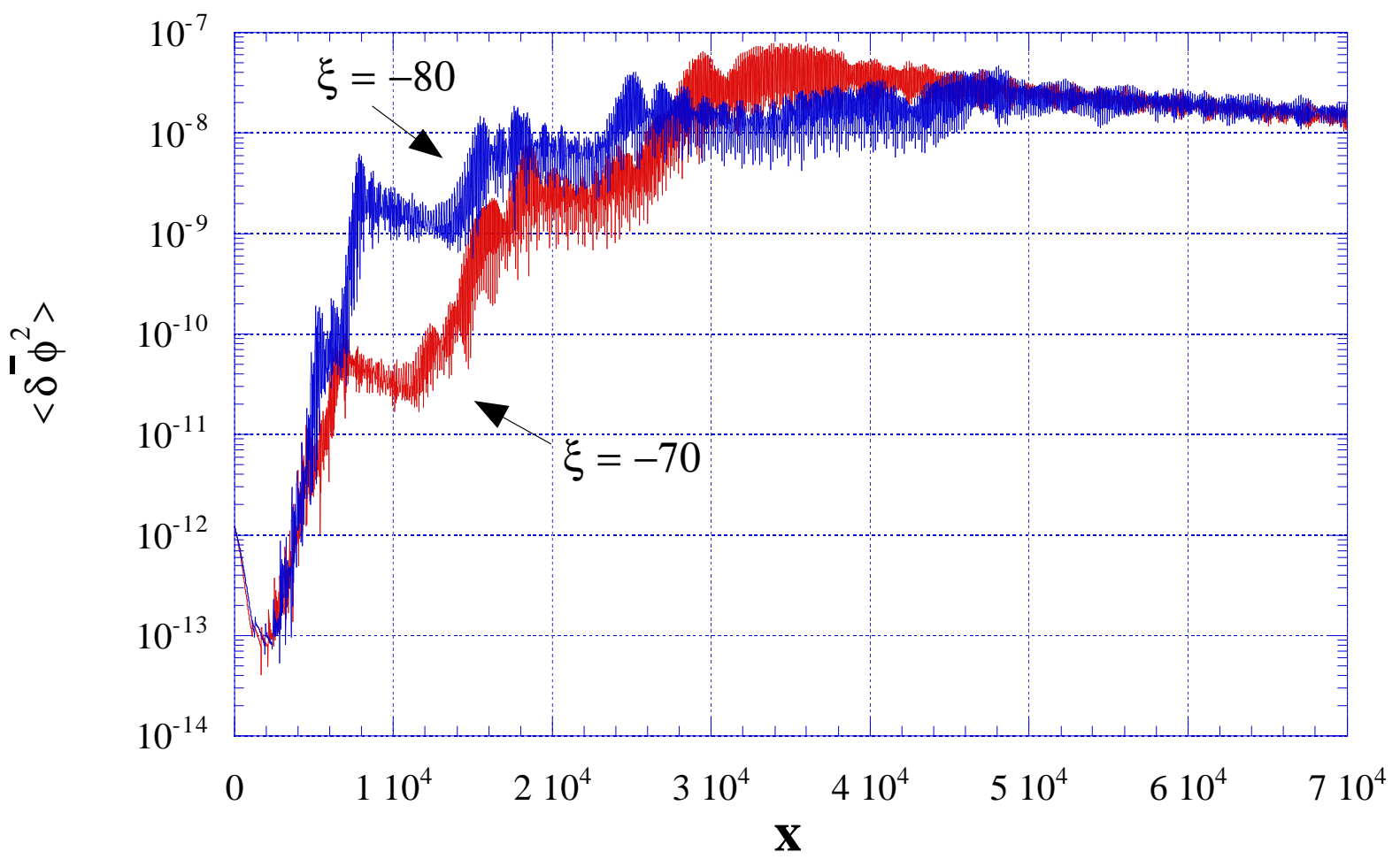


Fig. 8

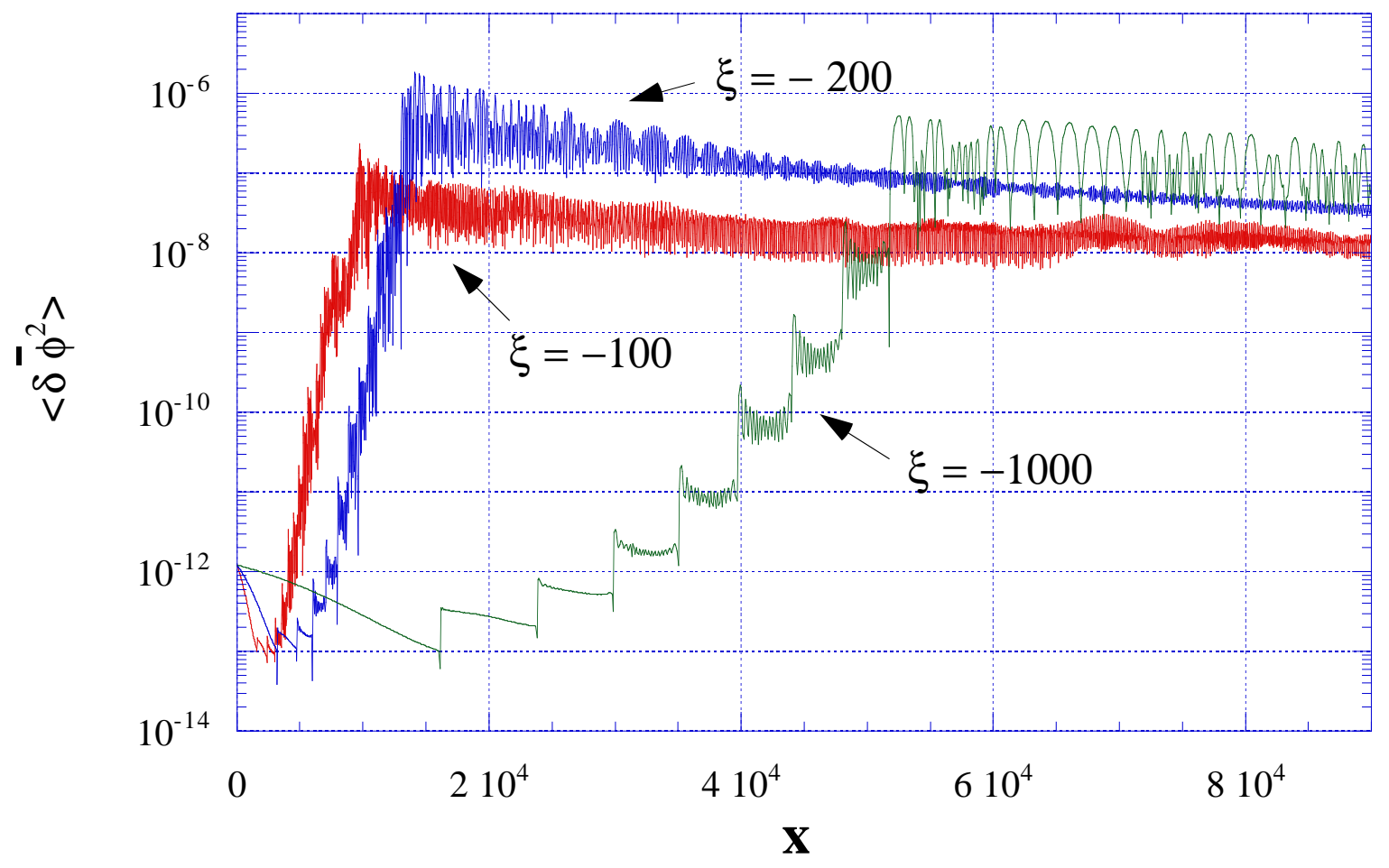


Fig. 9

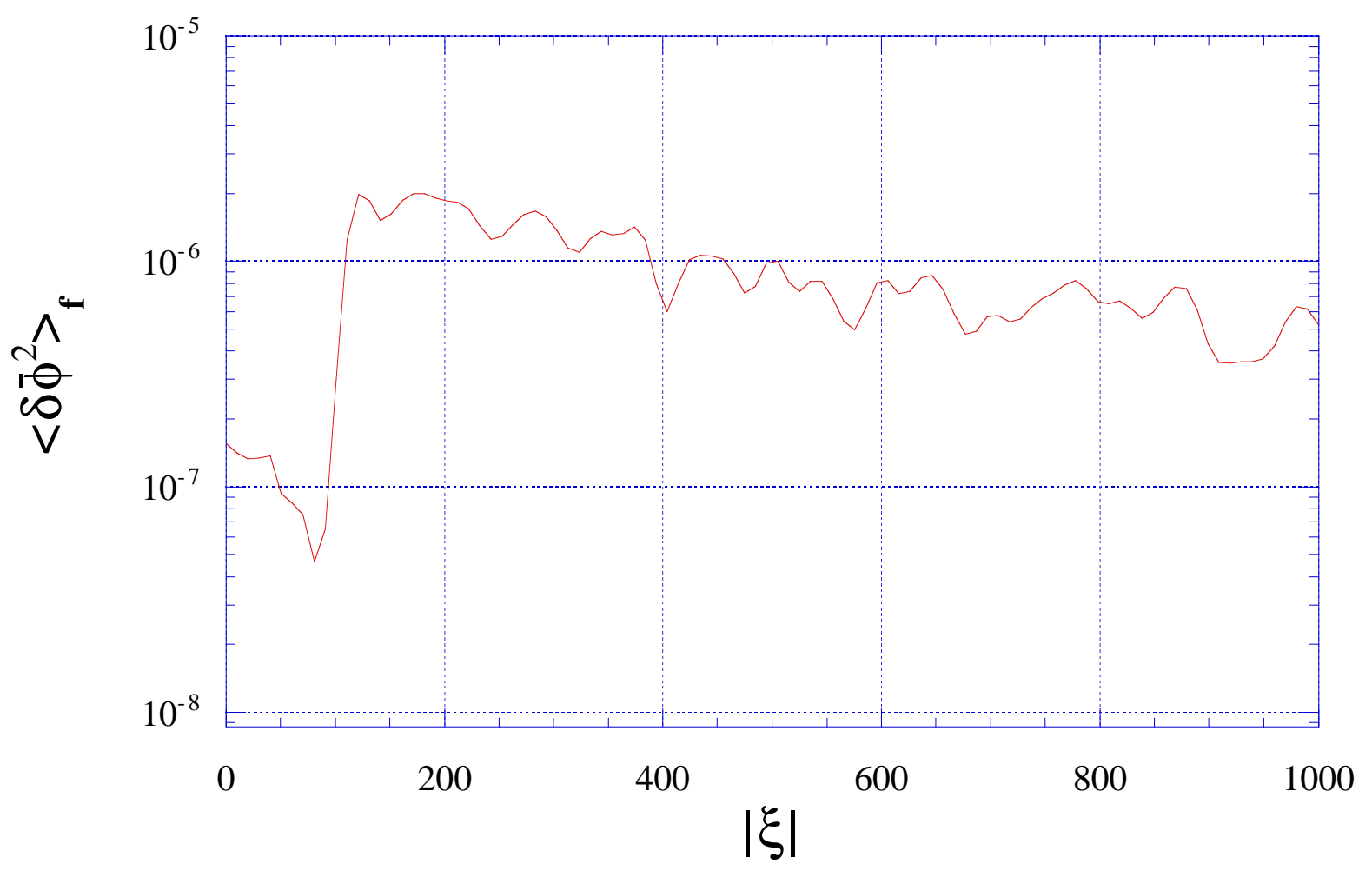


Fig. 10

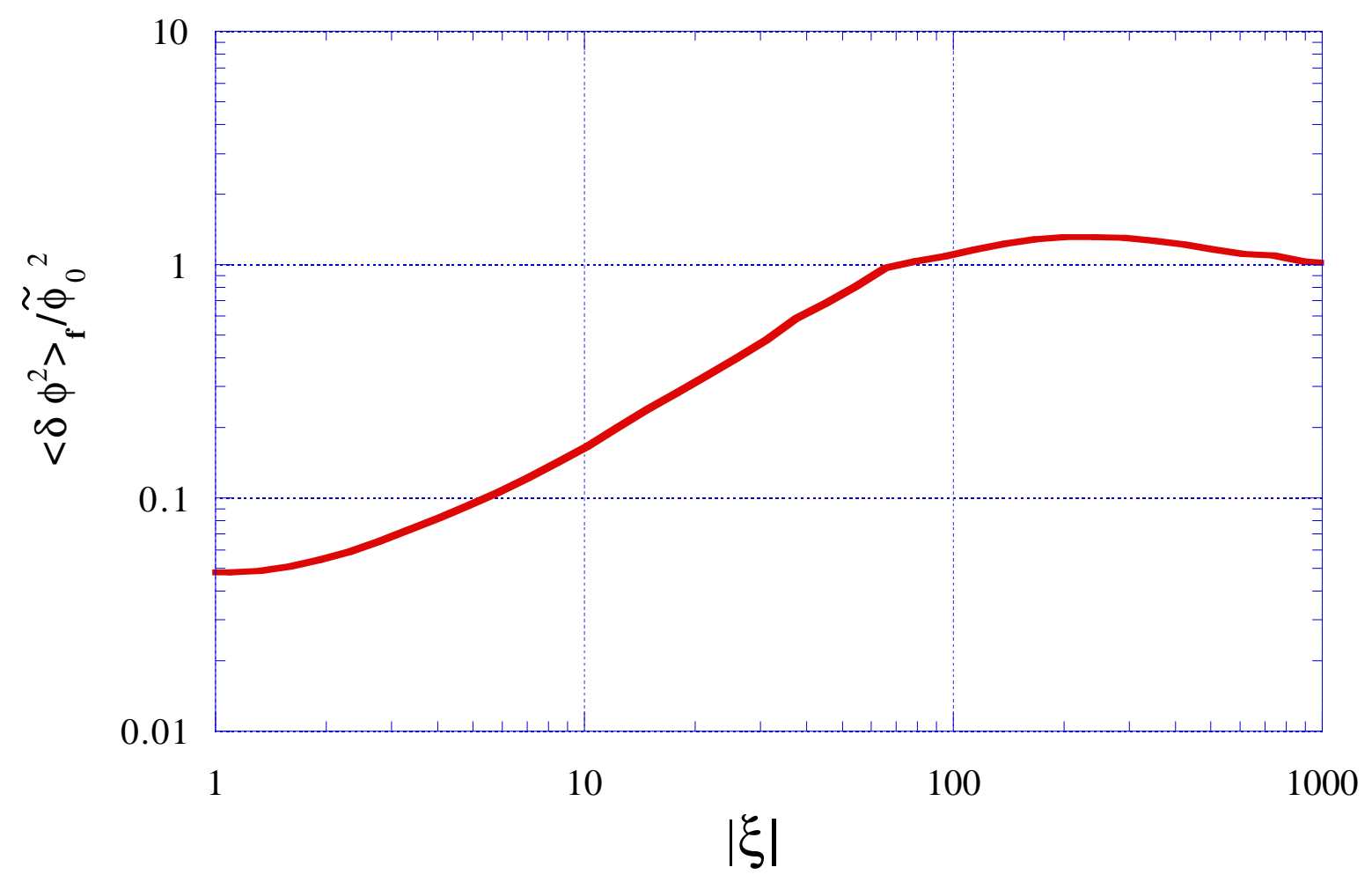


Fig. 11

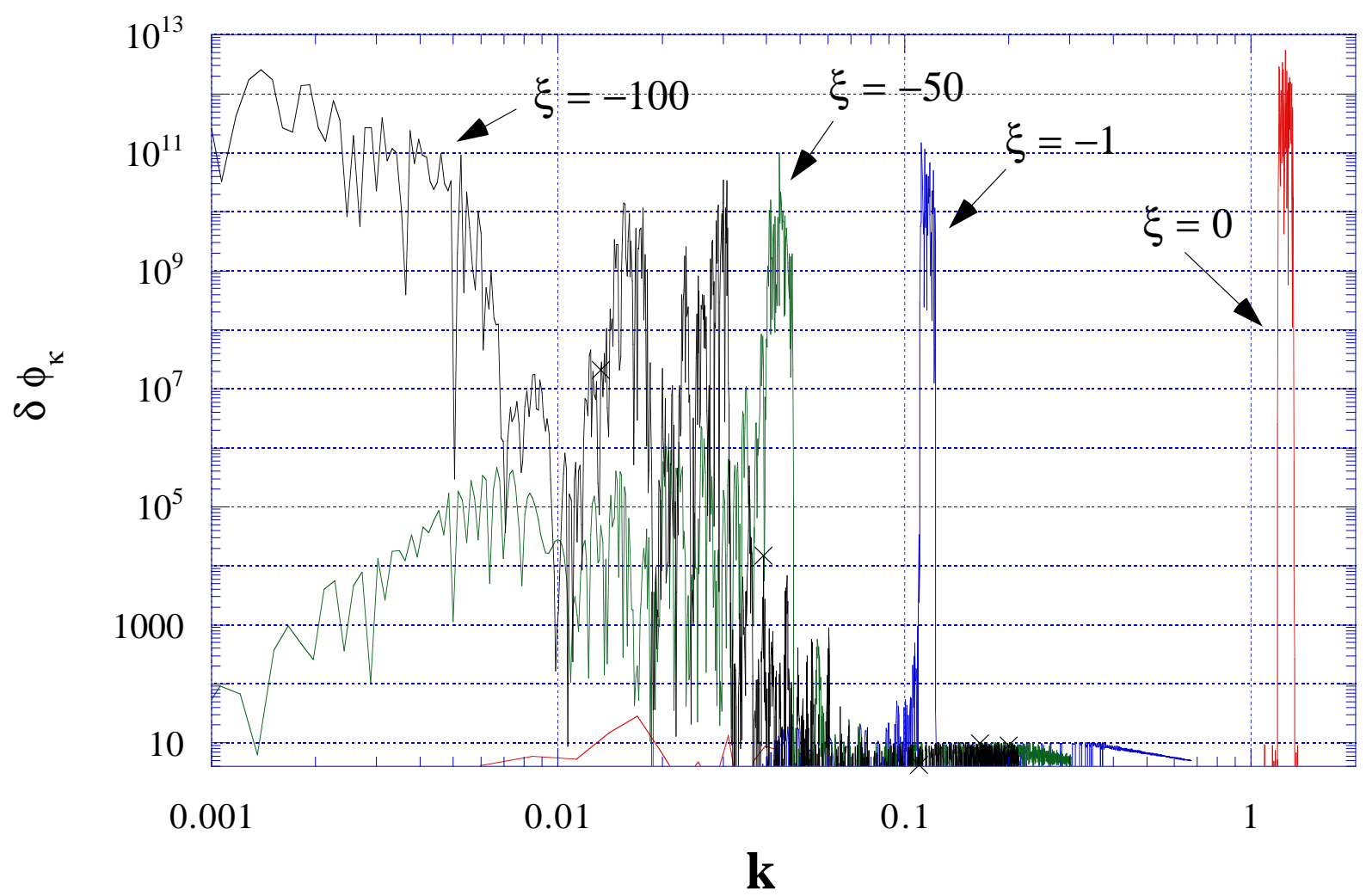

